\title{
FGFR4 regulates tumor subtype differentiation in luminal breast cancer and metastatic disease
}

\author{
Susana Garcia-Recio, ${ }^{1,2}$ Aatish Thennavan,, ${ }^{1,3}$ Michael P. East, ${ }^{4}$ Joel S. Parker, ${ }^{1,2}$ Juan M. Cejalvo, ${ }^{5,6}$ Joseph P. Garay, ${ }^{1,2}$ \\ Daniel P. Hollern, ${ }^{1,2}$ Xiaping He, ${ }^{1,2}$ Kevin R. Mott, ${ }^{1,2}$ Patricia Galván, ${ }^{5,6}$ Cheng Fan, ${ }^{1,2}$ Sara R. Selitsky, ${ }^{1}$ Alisha R. Coffey, \\ David Marron, ${ }^{1}$ Fara Brasó-Maristany, ${ }^{5,6}$ Octavio Burgués, ${ }^{7,8}$ Joan Albanell, ${ }^{7,9,10,11,12}$ Federico Rojo, ${ }^{7,9,13}$ Ana Lluch, ${ }^{7,9,14,15}$ \\ Eduardo Martinez de Dueñas, ${ }^{7,9,16}$ Jeffery M. Rosen, ${ }^{17}$ Gary L. Johnson, ${ }^{4}$ Lisa A. Carey, ${ }^{18}$ Aleix Prat, ${ }^{5,6,19}$ and Charles M. Perou ${ }^{1,2,20}$ \\ 'Lineberger Comprehensive Center and ${ }^{2}$ Department of Cenetics, School of Medicine, ${ }^{3}$ Oral and Craniofacial Biomedicine Program, School of Dentistry, and ${ }^{4}$ Department of Pharmacology, Department of \\ Medicine, School of Medicine, University of North Carolina, Chapel Hill, North Carolina, USA. ${ }^{5}$ Translational Genomics and Targeted Therapeutics in Oncology (IDIBAPS), Barcelona, Spain. ${ }^{6}$ Medical Oncology \\ Department, Hospital Clinic, Barcelona, Spain. 'TEICAM, Spanish Breast Cancer Group, Madrid, Spain. ${ }^{8}$ Department of Pathology, Hospital Clinico Universitario de Valencia, Valencia, Spain. ${ }^{9}$ Centro de \\ Investigación Biomédica en Red de Oncologia (CIBERONC-ISCIII), Madrid, Spain. ${ }^{10}$ IMIM Hospital del Mar Medical Research Institute, Barcelona, Spain. "Medical Oncology Department Hospital del Mar, \\ Barcelona, Spain. ${ }^{1}$ Universitat Pompeu Fabra, Barcelona, Spain. ${ }^{13}$ Fundación Jiménez Díaz, Madrid, Spain. ${ }^{1{ }^{4} H o s p i t a l ~ C l i ́ n i c o ~ U n i v e r s i t a r i o ~ d e ~ V a l e n c i a, ~ V a l e n c i a, ~ S p a i n . ~}{ }^{15}$ Biomedical Research Institute INCLIVA, \\ Universitat de València, Valencia, Spain. ${ }^{16}$ Hospital Provincial de Castellón, Castellón, Spain. ${ }^{17}$ Department of Molecular and Cellular Biology, Baylor College of Medicine, Houston, Texas, USA. ${ }^{18}$ Division of \\ Hematology-Oncology, Department of Medicine, School of Medicine, University of North Carolina, Chapel Hill, North Carolina, USA. ${ }^{19}$ SOLTI Breast Cancer Research Group, Barcelona, Spain. ${ }^{20}$ Department of \\ Pathology and Laboratory Medicine, University of North Carolina, Chapel Hill, North Carolina, USA.
}

Mechanisms driving tumor progression from less aggressive subtypes to more aggressive states represent key targets for therapy. We identified a subset of luminal A primary breast tumors that give rise to HER2-enriched (HER2E) subtype metastases, but remain clinically HER2 negative (cHER2-). By testing the unique genetic and transcriptomic features of these cases, we developed the hypothesis that FGFR4 likely participates in this subtype switching. To evaluate this, we developed 2 FGFR4 genomic signatures using a patient-derived xenograft (PDX) model treated with an FGFR4 inhibitor, which inhibited PDX growth in vivo. Bulk tumor gene expression analysis and single-cell RNA sequencing demonstrated that the inhibition of FGFR4 signaling caused molecular switching. In the Molecular Taxonomy of Breast Cancer International Consortium (METABRIC) breast cancer cohort, FGFR4-induced and FGFR4-repressed signatures each predicted overall survival. Additionally, the FGFR4-induced signature was an independent prognostic factor beyond subtype and stage. Supervised analysis of 77 primary tumors with paired metastases revealed that the FGFR4-induced signature was significantly higher in luminal/ER+ tumor metastases compared with their primaries. Finally, multivariate analysis demonstrated that the FGFR4induced signature also predicted site-specific metastasis for lung, liver, and brain, but not for bone or lymph nodes. These data identify a link between FGFR4-regulated genes and metastasis, suggesting treatment options for FGFR4-positive patients, whose high expression is not caused by mutation or amplification.

\section{Introduction}

Treating metastatic breast cancer patients has become increasingly complex, in part due to the large number of therapeutic options in the second- and third-line settings. Tumor heterogeneity imparts another key challenge for breast cancer therapy, especially in those tumors that become resistant to standard ther-

Conflict of interest: CMP is an equity stock holder, consultant, and Board of Directors member of BioClassifier LLC and GeneCentric Therapeutics. CMP and JSP are also listed as inventors on patent applications for the Breast Cancer PAM50 Subtyping assay. AP is a consultant/advisory board member for NanoString Technologies. AL is on the Advisory Board and consults for Novartis, Pfizer, Roche/Genentech, Eisai, and Celgene, and as a clinician is involved in clinical research managed by the Instituto de Investigación Sanitaria (INCLIVA) of Amgen, Astra Zeneca, Boehringer-Ingelheim, GSK, Novartis, Pfizer, Roche/Genentech, Eisai, Celgene, and Pierre Fabre. Copyright: ( 2020, American Society for Clinical Investigation.

Submitted: May 16, 2019; Accepted: June 17, 2020; Published: August 17, 2020.

Reference information: / Clin Invest. 2020;130(9):4871-4887.

https://doi.org/10.1172/JCl130323. apies in the metastatic setting. Human epidermal growth factor receptor 2 (HER2), estrogen receptor (ER), and progesterone receptor $(\mathrm{PR})$ are the main tumor targets in breast cancer and guide most treatment decisions; however, lack of these receptors or resistance to ER/PR or HER2 targeting are major therapeutic problems. Previous studies have shown that the expression of molecular markers currently used to determine anti-HER2 treatments and endocrine therapy do not tend to vary in the majority of patients between the primary and metastatic tumors (1). For this reason, additional molecular alterations need to be identified in order to better explain tumor progression changes occurring during the metastatic process.

Breast cancers can be molecularly classified into 5 main intrinsic subtypes (basal-like, luminal A, luminal B, HER2enriched [HER2E], and a normal-like group) $(2,3)$. We previously reported that the intrinsic molecular subtypes of breast cancer are also broadly maintained during metastatic progression; however, some luminal tumors are an exception (4). Existing data identify 
A TCGA patients
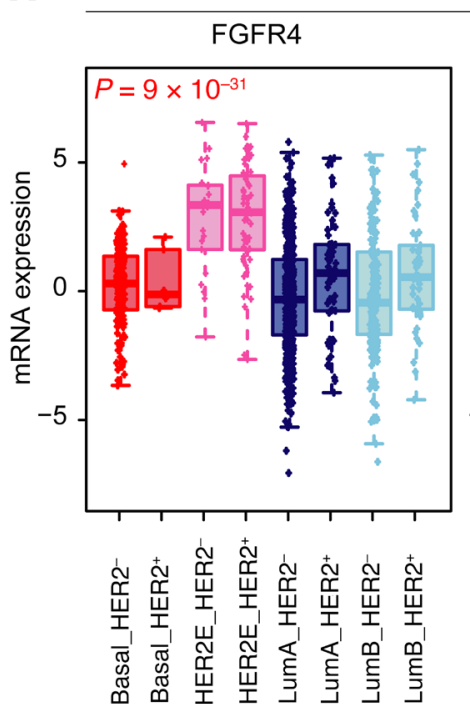
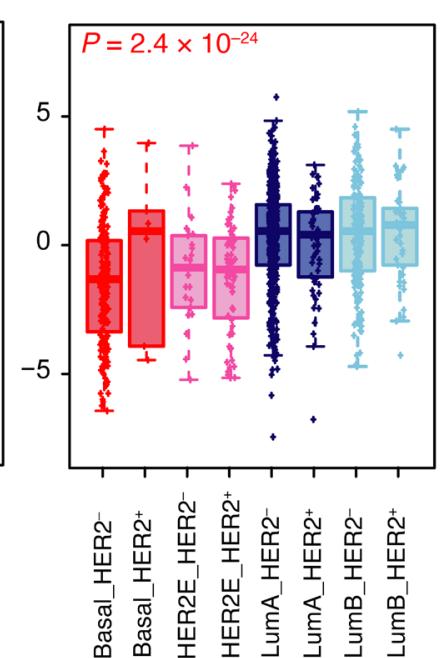

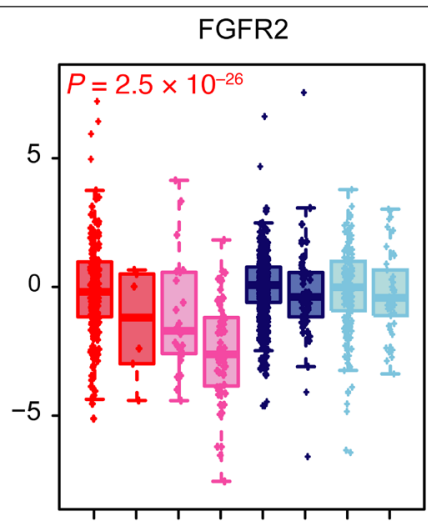

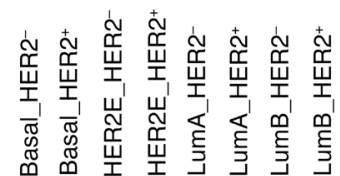

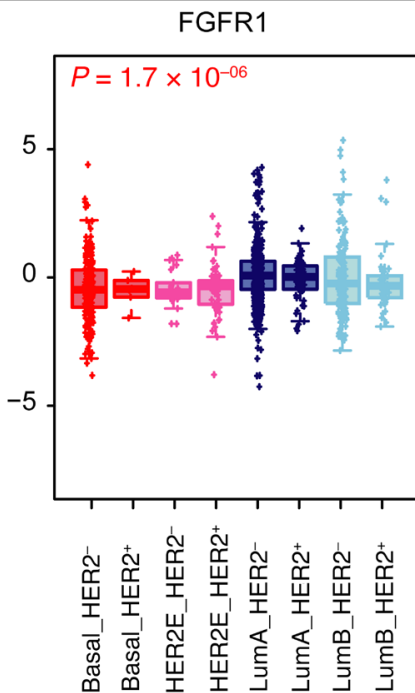

B

FGFR4*

FGFR4_Genetic alteration

HER2 status

PAM50 subtype

FGFR3*

FGFR3 Genetic alteration

FGFR2*

FGFR2_Genetic alteration

FGFR1*

FGFR1_Genetic alteration

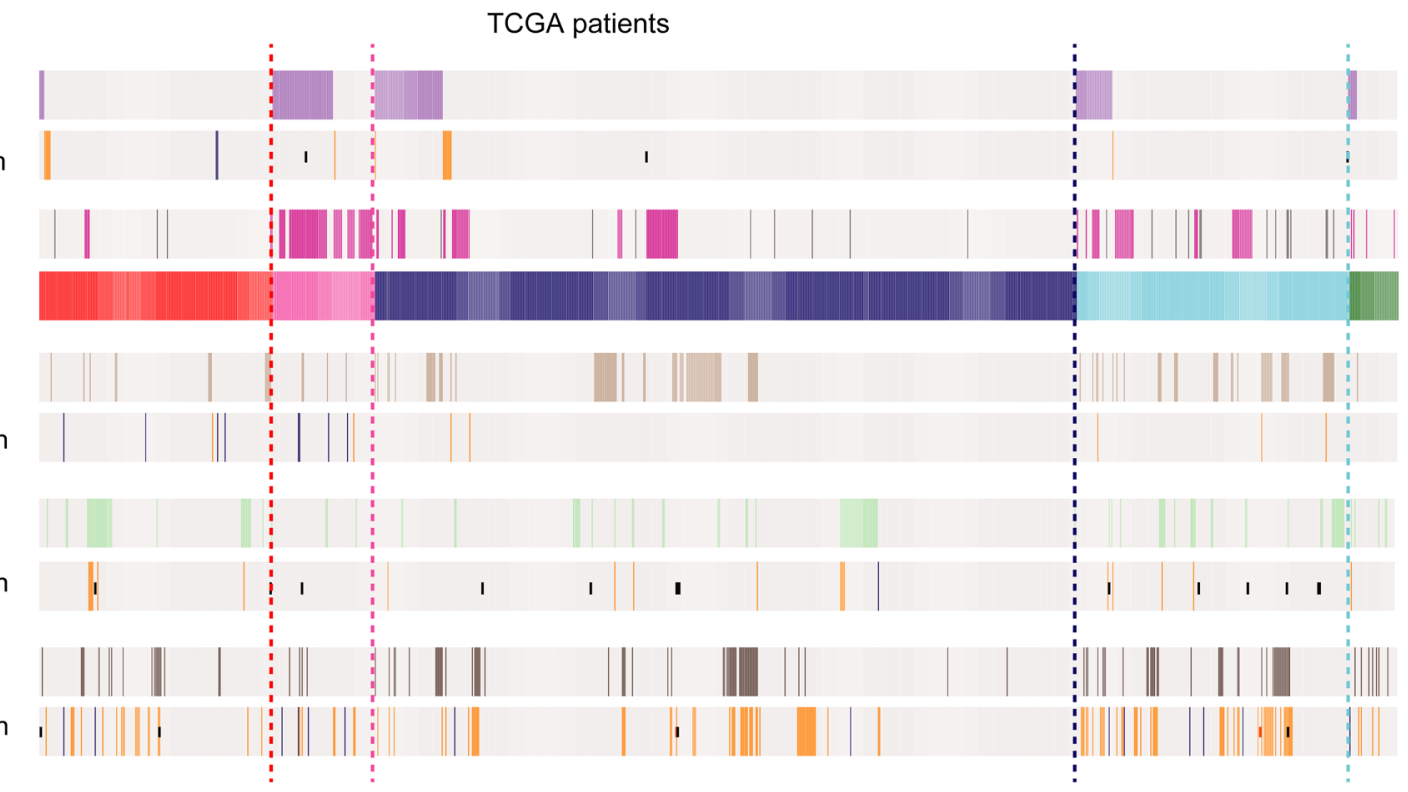

\begin{tabular}{l|l|l|l|l} 
PAM50 subtype & Basal & HER2-enriched $\mid$ Luminal A & Luminal B & Normal \\
HER2 status & Negative & Positive - N/A \\
Genetic alteration & No alterations & Amplification & Deep deletion
\end{tabular}

FGFR4 mRNA, high expression (Basal 2\%; HER2E59\%; LumA 10\%; LumB 13\%; Normal 18\%)

FGFR3 mRNA, high expression (Basal 7\%; HER2E5\%; LumA 15\%; LumB 21\%; Normal 3\%)

FGFR2 mRNA, high expression (Basal 18\%; HER2E5\%; LumA 9\%; LumB 14\%; Normal 13\%)

FGFR1 mRNA, high expression (Basal 10\%; HER2E5\%; LumA 10\%; LumB 18\%; Normal 2\%) 
Figure 1. Comparative genetic and transcriptomic analysis of FGFR4 family members in TCCA. (A) Box-and-whisker plots of FGFR family gene expression levels by mRNAseq according to molecular subtype and HER2 status by IHC. Tumors without clinical data for HER2 status and normal-like samples have been removed from the analysis, resulting in 1028 patients. Box-and-whisker plots display the median value on each bar, showing the lower and upper quartile range of the data and data outliers. The whiskers represent the interquartile range. Comparison between more than 2 groups was performed by ANOVA. Statistically significant values are highlighted in red. Each mark represents the value of a single sample. (B) Oncoprint diagram depicting high mRNA gene expression, DNA copy-number alterations, and mutations of FGFR family genes. ${ }^{*}$ FGFR mRNA, high expression: high expression of genes was considered where levels exceeded the third quartile of positive values of gene expression (normalized, $\log _{2}$-transformed, and median-centered RNAseq data). Putative copy-number calls on 1077 cases were determined using GISTIC 2.0 (84). Deep deletion: -2 (homozygous deletion); amplification: 2 (high-level amplification). Mutation types are defined only as missense mutations (single base pair) or truncating mutations (multiple base pairs). HER2 clinical status was defined as previously described (85). LumA, luminal A; LumB, luminal B.

a subset of primary luminal/ER $\mathrm{ER}^{+}$breast cancers that lose some of their luminal features and become more HER2E subtype-like in the metastatic setting $(4,5)$. This implies that the molecular drivers involved in this specific subtype switching may also represent crucial promoters of metastatic progression. The HER2E subtype can be divided into those that are clinically HER2 ${ }^{+}\left(\mathrm{cHER} 2^{+}\right)$or cHER2-, and also those that are $\mathrm{ER}^{+}$and ER', thus showing significant clinical heterogeneity within a single genomic subtype. Targeting HER2 is a landmark in the era of precision medicine and several drugs have been developed to treat $\mathrm{cHER} 2^{+}$tumors (6-9), but how best to treat the HER2E/cHER2- tumors is much less established. For this reason, we have been searching for possible druggable targets in HER2E/HER2- tumors. Within the HER2E subtype (including both cHER2- and cHER2 ${ }^{+}$, and $\mathrm{ER}^{+}$and $\mathrm{ER}^{-}$), fibroblast growth factor receptor 4 (FGFR4) is ubiquitously expressed and is a possible candidate driver gene of this subtype beyond HER2 itself (4). FGFR4 is a tyrosine kinase (TK) receptor involved in proliferation, survival, and migration during embryonic development. In adults, it contributes to tissue homeostasis, as well as tissue repair, angiogenesis, and inflammation $(10,11)$. FGFR4 is highly expressed in distinct cancer types (12-15) and also presents genetic variants such as the FGFR4 Arg388 polymorphism (16-19) in rare cases. Further, the molecular function and kinase domain of FGFR4 differs from other FGFRs, suggesting it may have a unique role when compared with them $(10,11)$. Despite these basic observations, the impact of FGFR4 on tumor progression in breast tumors remains poorly understood.

Importantly, we confirmed that FGFR4 is highly expressed in the HER2E subtype and in those tumors that acquire this HER2E state in the metastatic setting (4). As a result, we hypothesized that FGFR4 activation may represent a mechanism for subtype switching of a luminal primary tumor to a HER2E metastatic one. Here, we use therapeutic FGFR4 inhibition, genetic manipulations, protein kinome profiles, and single-cell RNA sequencing (scRNAseq) data to understand how FGFR4 alters molecular profiles, and to identify new possibilities for treatment options for primary and metastatic tumors in breast cancer.

\section{Results}

High FGFR 4 expression and the HER2E subtype. To test the relationship of FGFR signaling pathway activity to genomically defined intrinsic subtypes, we characterized the distributions of FGFR family members across all breast cancers and their somatic genetic alterations. We quantified mRNA expression of each FGFR family member (FGFR1-4) across all breast cancer subtypes in 3 independent data sets: The Cancer Genome Atlas (TCGA) (Figure 1A), METABRIC (Supplemental Figure 1A; supplemental material available online with this article; https://doi.org/10.1172/ JCI130323DS1), and MD Anderson Cancer Center (MDACC) (Supplemental Figure 1B). TCGA patients were classified into PAM50-defined subtypes and each subtype into 2 immunohistochemistry (IHC) subgroups according to clinical HER2 pathology report ( $\mathrm{cHER}^{+}$and $\left.\mathrm{cHER} 2^{-}\right)$. Next, we characterized the genetic and transcriptomic landscape of FGFR family members by subtype. FGFR4 mRNA expression was significantly higher in the HER2E subtype compared with FGFR1-3, and was independent of HER2 status, having high expression in both $\mathrm{cHER}^{+}$and $\mathrm{CHER}^{-}$ in all 3 data sets (Figure 1A and Supplemental Figure 1, A and B); FGFR4 was also mainly expressed in HER2E tumors. Conversely, FGFR2 and FGFR3 were mainly expressed in luminal/ER ${ }^{+}$tumors, and basal-like cancers showed low mRNA expression of all 4 FGFRs. TCGA data offered the opportunity to study the genetic alterations of breast cancer patients and we determined that the overexpression/high expression of FGFR4 is not driven by DNA copy-number status (Figure 1B); FGFR4 amplification/deletions and mutations were rare and were not correlated with high FGFR4 mRNA expression. Finally, we tested the mRNA levels of FGFR4 in normal breast tissue using TCGA data. FGFR4 expression levels were significantly higher in all tumor molecular subtypes compared with normal breast samples (Supplemental Figure 1C). Taken together, these data demonstrate that FGFR4 is unique among FGFR family members in its apparently nongenetically determined expression pattern across breast cancer subtypes, with high expression in HER2E genomic subtype tumors.

FGFR4 inhibitor treatment of a HER2E/cHER2- patient-derived xenograft. To determine if FGFR4 is a driver in HER2E-subtype tumors, we tested 2 possible druggable targets in HER2E/cHER2tumors, namely FGFR4 itself and HER2. To model human breast cancer, we used NSG mice bearing an endocrine-resistant FGFR4 ${ }^{+}$ patient-derived xenograft (PDX) model called WHIM11. WHIM11 is of the HER2E subtype and was originally described as cHER2-, EGFR', and $\mathrm{ER}^{+}$(Supplemental Figure 2, A and B, and Supplemental Table 1) and thus reflects those cases of human breast cancer where FGFR4 is highly expressed in a nongenetic fashion $(20,21)$. However, we observed some HER2 protein expression in this model based on IHC assay using anti-HER2 CB11 antibody (the FDA-approved antibody for HER2 detection) compared with other PDXs considered cHER2 ${ }^{+}$(WHIM35 and WHIM8) or cHER2- (WHIM30 and WHIM2) (Supplemental Figure 2B). To target FGFR4 or HER2, we used a selective and irreversible FGFR4 inhibitor (BLU9931) (22), and a dual reversible inhibitor of HER2 and EGFR (lapatinib) (23); we note here that the efficacy of lapatinib in a group of patients with HER2E/cHER2- disease has been demonstrated via retrospective analysis (24). The doses of BLU9931 assessed were 0.3 and $0.6 \mathrm{~g} / \mathrm{kg} / \mathrm{day}$ as previously 
A

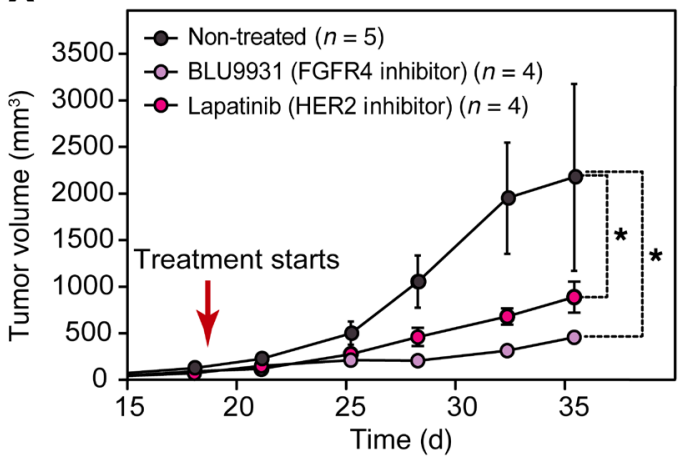

B

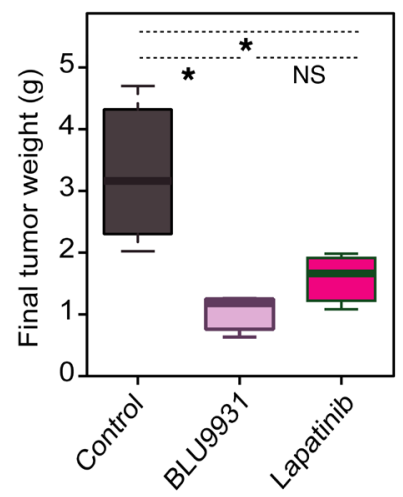

C

More similar to the subtype
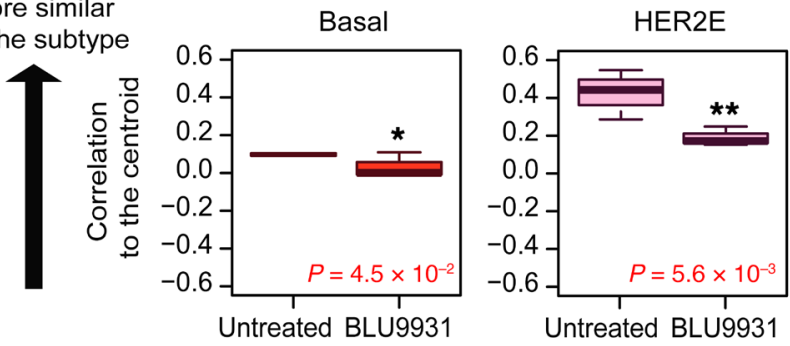

More similar to the subtype
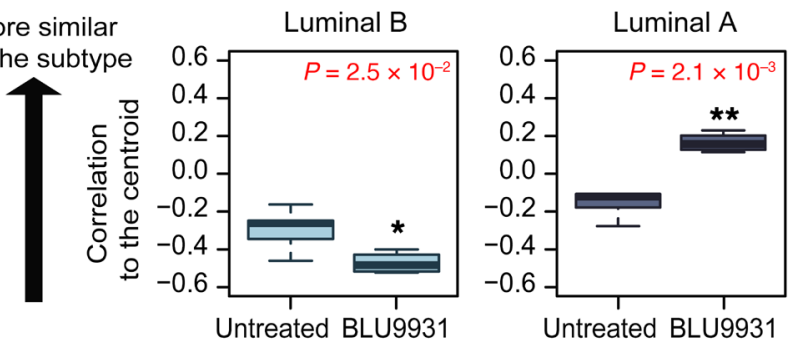

$\mathbf{E}$

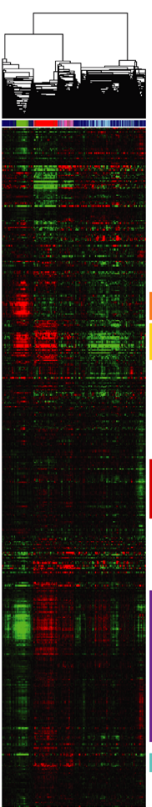

FGFR4-induced signature SAM DOWN regulated genes

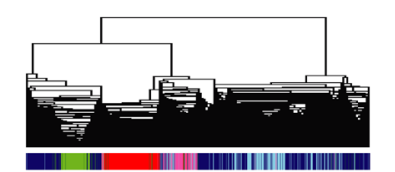

- HER2E

LumB

- LumA

- Normal-like

normal tissue

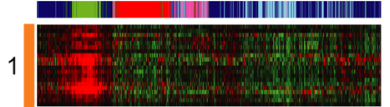

Ligand-binding receptor protein phosphorylation

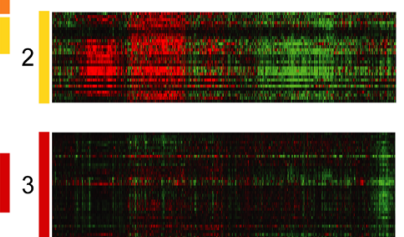

Tissue epithelium development

Protein localization and transport

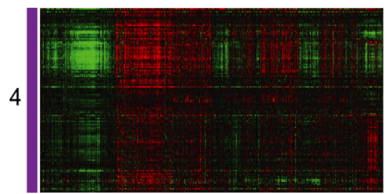

Cell cycle and proliferatio

5

Monocarboxylic acids or oxidation-reduction processes
D

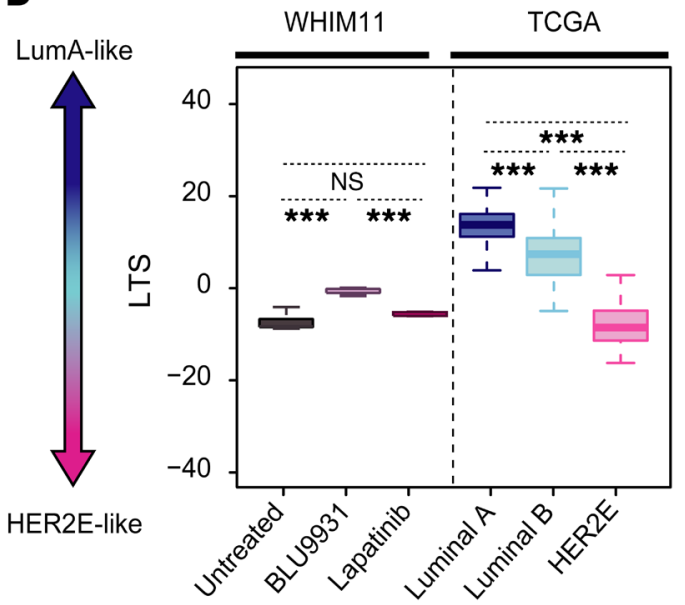

SAM UP regulated genes

Basal

- HER2E

LumB

- LumA

Normal-like

- Normal tissue
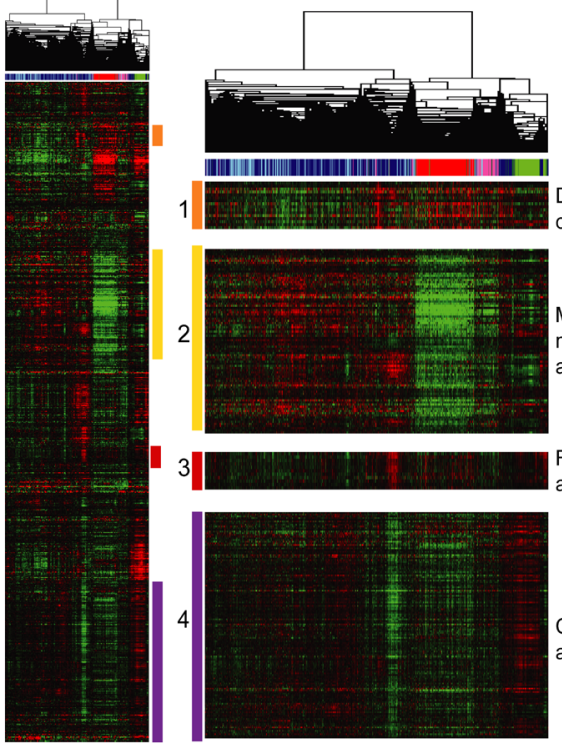

Development or functioning

of the immune system

Mammary gland development, morphogenesis of epithelial tubes and estrogen response signaling

Chromatin modification and regulation of transcription 
Figure 2. Testing the impact of FGFR4 on tumor growth and tumor differentiation in a HER2E/cHER2- PDX (WHIM11). (A) Tumor growth and (B) tumor weight of WHIM11 tumors treated with BLU9931 (0.6 g/ kg/day) or lapatinib $(1.833 \mathrm{~g} / \mathrm{kg} /$ day) for approximately 18 days ( 5 animals per treatment arm). Data represent mean tumor volume \pm SEM. (C) Correlation to the PAM50 centroids (basal, HER2E, luminal A, luminal B) of mice treated with BLU9931 and untreated mice. (D) BLU9931-treated, lapatinib-treated, or untreated tumors evaluated for a luminal tumor score (LTS) tested along with TCCA cohort grouped according to intrinsic subtype. (E and $\mathbf{F}$ ) Supervised hierarchical cluster across 1100 breast tumors and 98 normal samples from TCGA data set using FGFR4-related signatures. Significantly upregulated genes defined as an FGFR4repressed signature $(\mathbf{E})$ and significantly downregulated genes defined as a FGFR4-induced signature (F). Each gene set or subcluster has been selected based on a node correlation greater than 0.5 and named according to the important biological process governed by it as identified by Gene Ontology. Comparison between more than 2 groups was performed by ANOVA with post hoc Tukey's test: ${ }^{*} P<0.05$; ${ }^{* *} P<0.01$; ${ }^{* * *} P<0.001$. Statistically significant values are highlighted in red. Box-and-whisker plots display the median value on each bar, showing the lower and upper quartile range of the data. The whiskers represent the interquartile range.

described (22) for a period of 4 weeks; we observed that neither dose induced weight loss or other signs of significant toxicity, and we thus treated the mice with $0.6 \mathrm{~g} / \mathrm{kg} / \mathrm{day}$. For lapatinib, we used the same dose $(1.833 \mathrm{~g} / \mathrm{kg} /$ day $)$ that we previously described (20, $25,26)$. Tumor volume (Figure 2A) and tumor weight (Figure 2B) were significantly reduced in WHIM11 tumor-bearing mice treated with BLU9931 or lapatinib (all $P<0.01$ ) for 18 days.

FGFR 4 as a potential driver of tumor dedifferentiation. We and others have hypothesized that the intrinsic subtypes of breast cancer recapitulate the normal breast epithelial differentiation hierarchy axis (mammary stem cell $\rightarrow$ luminal progenitor cells $\rightarrow$ mature luminal cells) $(27,28)$. We previously developed a transcriptome-defined differentiation score based on purified mammary epithelial cell populations (29). The intrinsic molecular subtypes can be ordered according to their differentiation score where the claudin-low-subtype tumors are the least differentiated and most like mammary stem cells, followed by basal-like tumors that are the most like luminal progenitor-like, followed by HER2E and then luminal A/B that are the most differentiated and closest to mature luminal cells (29). We hypothesized that FGFR4 could be an important driver of shifts between subtypes and among this differentiation hierarchy. To test this, we collected gene expression data of untreated and BLU9931-treated WHIM11 tumors and calculated the correlation of each to the PAM50 subtype centroids (30). WHIM11 tumors treated with BLU9931 were significantly more similar to the luminal A subtype and less HER2E, luminal B, or basal-like compared with the control group (Figure 2C). Although lapatinib treatment significantly decreased the tumor growth of WHIM11, no significant change was found by comparing the PAM50 centroids before and after treatment (Supplemental Figure 3A), showing that inhibition of FGFR4 activity affected subtype differentiation status in this model, while HER2 inhibition did not.

Next, in order to find a better alignment with tumor-intrinsic subtype differentiation status, we developed a transcriptional phenotype score based on similarity to tumor subtypes (as opposed to similarity to normal epithelial cells for the aforementioned differ- entiation score); we constructed a model having luminal A versus HER2E as the axis of separation, and call this the "luminal tumor score" (LTS). This method allows us to calculate a relative score of segregation between luminal A and HER2E regardless of the experimental platform or models used (see Methods). The LTS is based on TGCA breast cancer data and compared with untreated and treated WHIM11 tumors (BLU9931 or lapatinib); WHIM11 tumors treated with BLU9931 showed a significantly higher LTS (more luminal-like) compared with the untreated $(P<0.001)$ or lapatinib-treated $(P<0.001)$ tumors (more HER2E-like) (Figure $2 D)$. This result suggests that genes initially repressed by the activity of FGFR4 were those related to luminal tumor phenotypes.

Characterization of FGFR4-derived gene expression signatures. To further characterize FGFR4 activity, we compared gene expression profiles between control and BLU9931-treated WHIM11 tumors. We performed a 2-class significance analysis of microarrays (SAM) (31) using a false discovery rate (FDR) of $1 \%$ $(32,33)$. This identified 745 upregulated genes that were initially repressed by the activity of FGFR4 before BLU9931 treatment (referred to hereafter as the FGFR4-repressed signature), and 427 downregulated genes that were positively regulated by FGFR4 activity before treatment (FGFR4-induced signature). The FGFR4-induced signature contained proliferation genes such as E2F1, CCNB2, CDC6, ORC6, and POLE2, survival-related genes including MET and PRKCA, genes related to epithelial-mesenchymal transition (EMT) including ITGB5, CXCL1, and COL4A2, and genes involved in subtype differentiation such as KRT19, KRT16, and ITGA6 (also known as CD49f) (Supplemental Table 2). Conversely, the FGFR4-repressed signature contained many known luminal subtype-related genes also involved in mammary gland development such as ESR1, FOXA1, GBLI3, PRLR, BCL2, and ERBB4 (Supplemental Table 2).

Next, each FGFR4-associated gene set was hierarchically clustered across 1100 breast tumors and 98 normal samples from TCGA data set (Figure 2, E and F) representing all 5 subtypes of breast cancer. We identified multiple distinct gene clusters and by examining Gene Ontology (GO) we considered each cluster to represent a different FGFR4-associated biological signature. Gene cluster associations with GO for the FGFR4-induced signature were as follows: ligand-binding receptor and protein phosphorylation (cluster 1), tissue epithelium development (cluster 2), protein localization and transport (cluster 3), cell cycle and proliferation (cluster 4), and chemical reactions and pathways involving monocarboxylic acids or oxidation-reduction processes (cluster 5) (Figure 2E and Supplemental Table 3). In order to differentiate changes derived from FGFR4 activity from those directly related to inhibition of cell proliferation caused by FGFR4 inhibition, the gene set known to be associated with proliferation (34-36) was removed from the FGFR4-induced signature from this point forward (cluster 4). Hallmark gene set enrichment analysis (34) of the entire-gene FGFR4-induced signature list revealed that this was enriched for genes involved in STAT3, PI3K/AKT/mTOR pathway, and KRAS activation, hypoxia, glycolysis, EMT, and genes encoding components of apical junctions (Supplemental Table 5).

Clustering of the FGFR4-repressed signature genes revealed 4 unique clusters. GO analysis revealed that these genes were related to development or immune system function (cluster 1); 
A

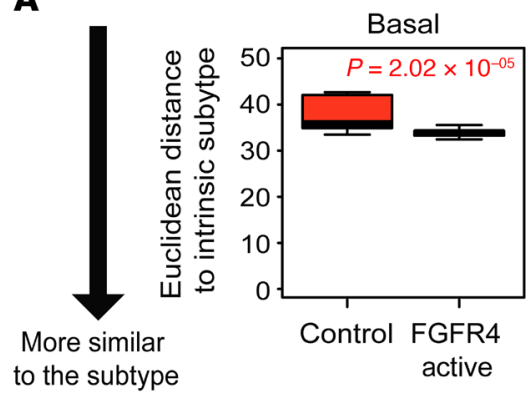

HER2E

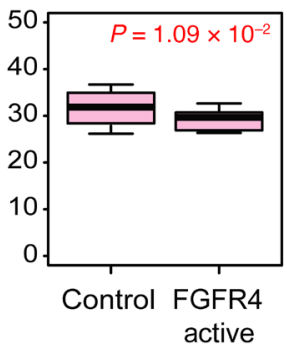

Luminal A

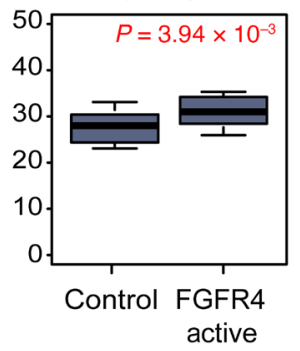

Luminal $B$

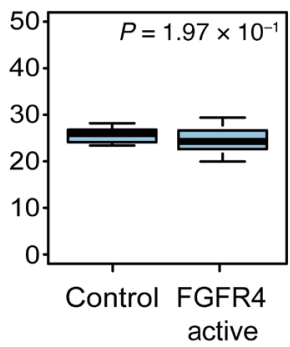

\section{B}

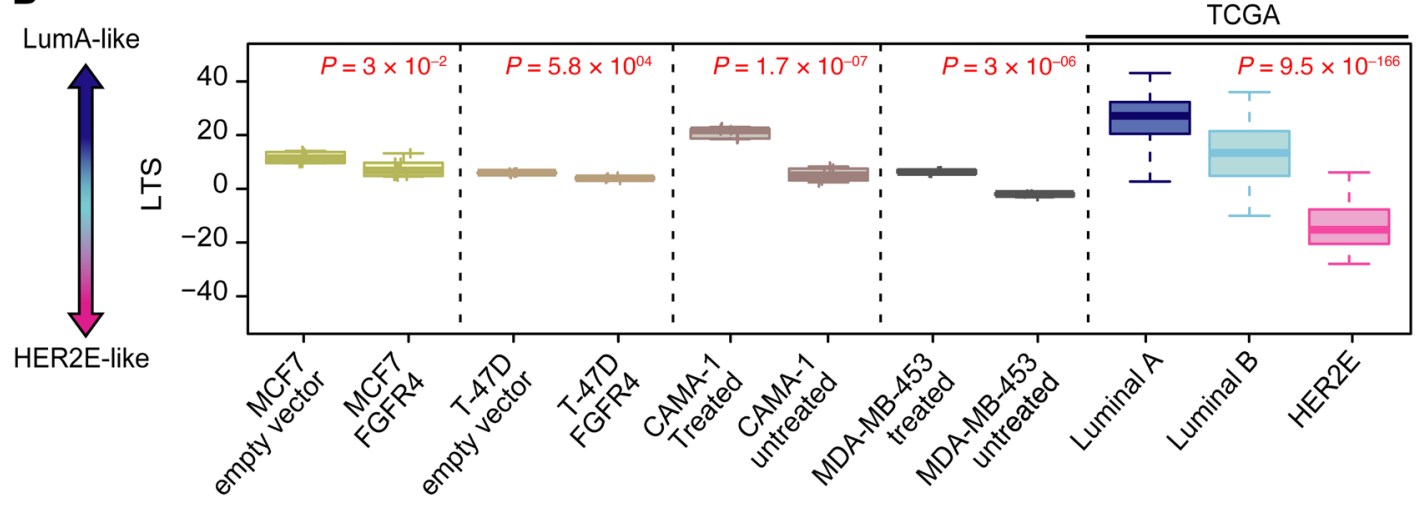

C

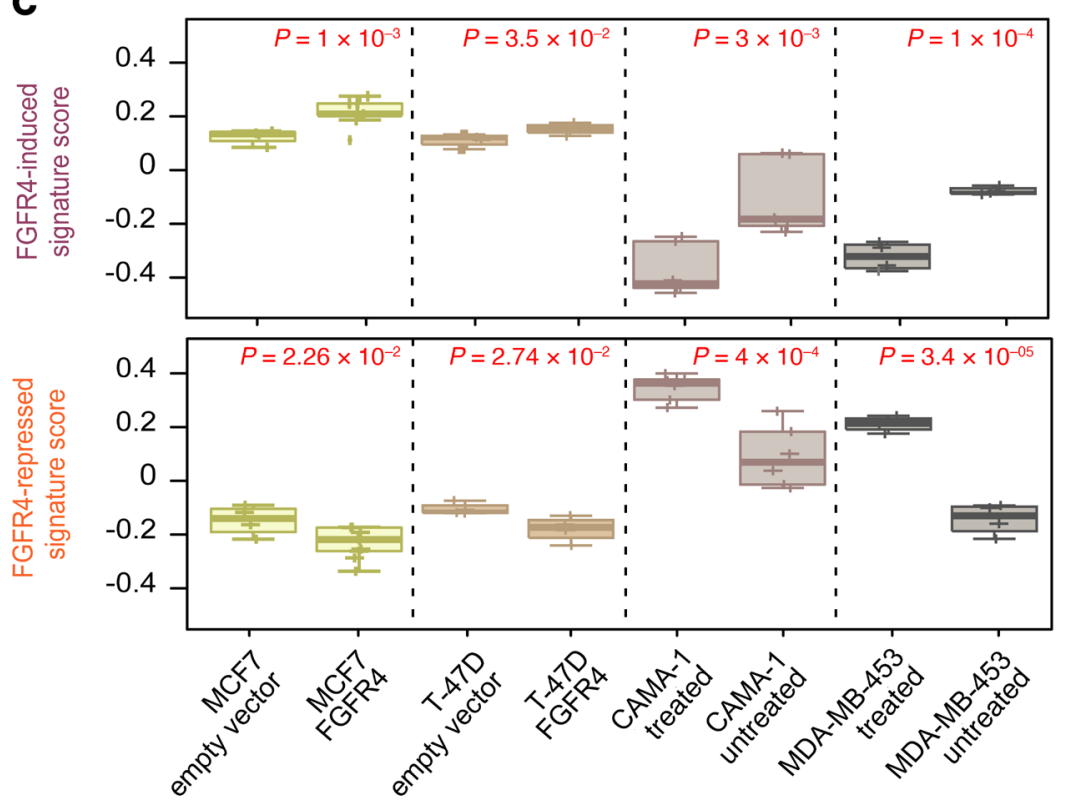

Figure 3. Testing FGFR4-associated signatures in genetically engineered or chemically inhibited breast cancer cell lines. (A) Box-and-whisker plots depicting the Euclidean distance of each group of cell lines to the UNC337 tumor-intrinsic subtypes. Lower Euclidean distance suggests higher similarity to the subtype call. (B) Analysis of luminal tumor score (LTS) in each cell line tested along with TCGA grouped according to intrinsic subtype. (C) Average expression (signature score) of FGFR4-induced signature and FGFR4-repressed signature of each cell line described in $\mathbf{A}$ and $\mathbf{B}$. Comparisons between 2 groups were performed by 2-tailed $t$ test. Comparison between more than 2 groups was performed by ANOVA. Box-and-whisker plots display the median value on each bar, showing the lower and upper quartile range of the data and data outliers. The whiskers represent the interquartile range. Each mark represents the value of a single sample. Statistically significant values are highlighted in red.

mammary gland development, morphogenesis of epithelial tubes, and estrogen response signaling (cluster 2); ribosome-related genes and protein targeting (cluster 3); and chromatin modification and regulation of transcription (cluster 4) (Figure $2 \mathrm{~F}$ and
Supplemental Table 4). Hallmark gene set enrichment analysis (34) of the entire gene list showed that this signature was enriched for genes involved in bile acid metabolism, genes downregulated by KRAS signaling, genes overrepresented on the apical surface 
important for cell polarity, genes related to the p53 pathway, genes related to peroxisomes, and genes upregulated in response to IFN- $\gamma$ and TGF- $\beta$ (Supplemental Table 5).

Proteomic profiling of the FGFR4 kinome in a PDX. To further characterize the role of FGFR4 in WHIM11, we quantitatively measured the dynamic changes in kinase activity using multiplexed inhibitor beads coupled with mass spectrometry (MIB/ MS) (37-39). We profiled the kinome of WHIM11 tumors treated with BLU9931 for 18 days and then released from this inhibition for 14 days, to identify those kinases that were induced upon removal of the drug; 258 kinases were detected in total. We next used 2-class SAM (31) with an FDR of $5 \%$ to identify proteins with significantly different levels (Supplemental Table 6); after BLU9931 treatment, 41 kinases were significantly reduced and 54 were upregulated where FGFR4 was the most significantly repressed kinase (Supplemental Table 6). After drug removal, 28 kinases were significantly induced and 46 repressed, and again FGFR4 had the most dramatically changing protein levels (Supplemental Table 6). These protein data were hierarchically clustered, and as expected, a significant portion of these patterns was associated with proliferation and decreased with treatment and induced upon drug removal (Supplemental Figure 4A). The 51 commonly affected kinases after BLU9931 treatment and expressed again after drug removal describe the reactivation of the FGFR4 pathway and are represented in Supplemental Figure $4 \mathrm{~B}$, grouped by 11 different families of kinases. In addition to FGFR4, the main induced kinases after drug removal were proliferation-related groups including NEK (NEK2), CAMK group (MELK), CMCG group (CDK1 and CDK13), and the Aurora family (AURKA and AURKB). Other induced kinases included some involved in mRNA splicing from the CMCG group (SRPK1 and CLK1) and some involved in controlling growth and differentiation like the ACG group (RPS6KA4, RPS6KA1, and RPS6KA3). Importantly, this result shows how BLU9931 dampened FGFR4 activity while other TK-family receptors remained relatively unchanged (only EPHA2 decreased in a small proportion), thus demonstrating that BLU9931 exhibited limited off-target effects on the TK-family receptors analyzed (Supplemental Figure 4B). Finally, we note that a portion of these kinases (CHUK, MELK, ZAK, RPS6KA4, RPS6KA3, PLK1, PLK4, AURKB, PKMYT1, HK2, and PRKAA2) were also identified in our gene expression analysis of FGFR4 signaling in WHIM11 tumors (Supplemental Figure 4C and Supplemental Table 6).

Validation of FGFR4-associated signatures in breast cancer cell lines. To test if ectopic expression/activation of FGFR4 alters differentiation status in established breast cancer cell lines, we used lentiviral particles containing the FGFR4 gene to transduce 2 luminal breast cancer cell lines, MCF7 and T47D (both HER2-FGFR $4^{\text {lo }} \mathrm{ER}^{+}$; see Methods section, Supplemental Table 1, and Supplemental Figure 6, A and B for FGFR4 protein quantification). In addition, we obtained 2 cell lines with high levels of FGFR4 activity: the MDA-MB-453 luminal cell line (FGFR $4{ }^{\text {hi }} \mathrm{HER}^{+} \mathrm{ER}^{-}$) that has a known activating mutation in FGFR4 (40) and the luminal cell line CAMA-1 (FGFR4 ${ }^{+}$HER2- ${ }^{-} R^{+}$) (Supplemental Figure 5A). The experimental conditions for gene expression analysis are summarized in Supplemental Figure 5, A and B (at least 4 replicates for each cell line). We treated the FGFR4-active cell lines (MDA-
MB-453 and CAMA-1) with BLU9931 at their IC $_{50}$ doses (Supplemental Figure 6C) to identify FGFR4-regulated genes, and at the same time identified the sets of genes induced upon introduction of FGFR4 into the $2 \mathrm{FGFR}^{\text {lo }}$ luminal/ER ${ }^{+}$cell lines (MCF7 and T47D). The overall gene expression patterns of each cell line was then compared to each PAM50 tumor subtype using the Euclidean distance to each PAM50 subtype centroid, which showed that the FGFR4-active group (i.e., CAMA-1 and MDA-MB-453 without drug, and MCF7 and T47D + FGFR4) were more similar to HER2E and/or basal-like tumors and less similar to luminal A tumors (Figure 3A). Likewise, cells overexpressing FGFR4 showed a significantly lower LTS (more HER2E-like), and by treating those cells with BLU9931, they gained a significantly higher LTS (more luminal-like) while losing their HER2E features (Figure 3B).

We also tested the WHIM11-derived FGFR4 signatures (induced and repressed). As expected, the FGFR4-activated cell lines had higher expression of the FGFR4-induced signature, and conversely, the FGFR4-repressed signature was enriched in cell lines with blocked FGFR4 activity (Figure 3C). Given recent reports that FGFR4 may impact endocrine resistance (41), we tested if expressing high levels of FGFR4 could lead to estrogenindependent growth using 2 luminal/ER $\mathrm{ER}^{+}$breast cancer cell lines. MCF7-FGFR4 $^{+}$and T47D-FGFR4 ${ }^{+}$cells presented higher cell viability compared with empty vector-transfected controls after 6 days of estrogen deprivation (Supplemental Figure 6E). Thus, FGFR4 activation may facilitate departure from luminal-like programming toward HER2E by facilitating independence from hormone receptor signaling.

As a second FGFR4-targeting approach, we used the CRISPR genome editing system to genetically inactivate FGFR4; however, we performed CRISPR/Cas9 on MDA-MB-453 and CAMA-1 cell lines and we were not able to obtain a homozygous knockout clone. According to the estimation of cancer gene dependencies from a large-scale RNAi screen published by McFarland et al. (42), FGFR4 is an essential gene in MDA-MB-453 and CAMA-1 cell lines measured by the DEMETER2 method (42). MDA-MB-453 and CAMA-1 cell lines have one of the highest FGFR4 gene dependency scores compared with a panel of breast cancer cell lines, in accordance with a higher FGFR4 gene expression (Supplemental Figure 6D), which we believe explains the inability to obtain CRISPR double-knockout clones.

Analysis of subtype switching using scRNAseq on WHIM11. To precisely determine if the shift between subtypes represents an example of cellular plasticity under the selective pressure of BLU9931 treatment, or alternatively is due to clonal selection, we performed 2 different genomic experiments. First, we used whole-exome sequencing to compare DNA markers of clonality in untreated and BLU9931-treated WHIM11 tumors that were allowed to regrow after removal of BLU9931 for 2 weeks. A supervised analysis using the 31 mutations described in WHIM11 in Li et al. (ref. 21 and Supplemental Table 7) showed a very high correlation between the variant allele frequency (VAF) of untreated WHIM11 tumors and BLU9931-treated and released tumors (Pearson's $r=0.97, P<0.001$ ) (Supplemental Figure 7A). Similar results were found when we compared our WHIM11 tumors with the original WHIM11 from Washington University published in 2013 (21) (Pearson's $r=0.83, P<0.001$ ) (Supplemental Figure 
A

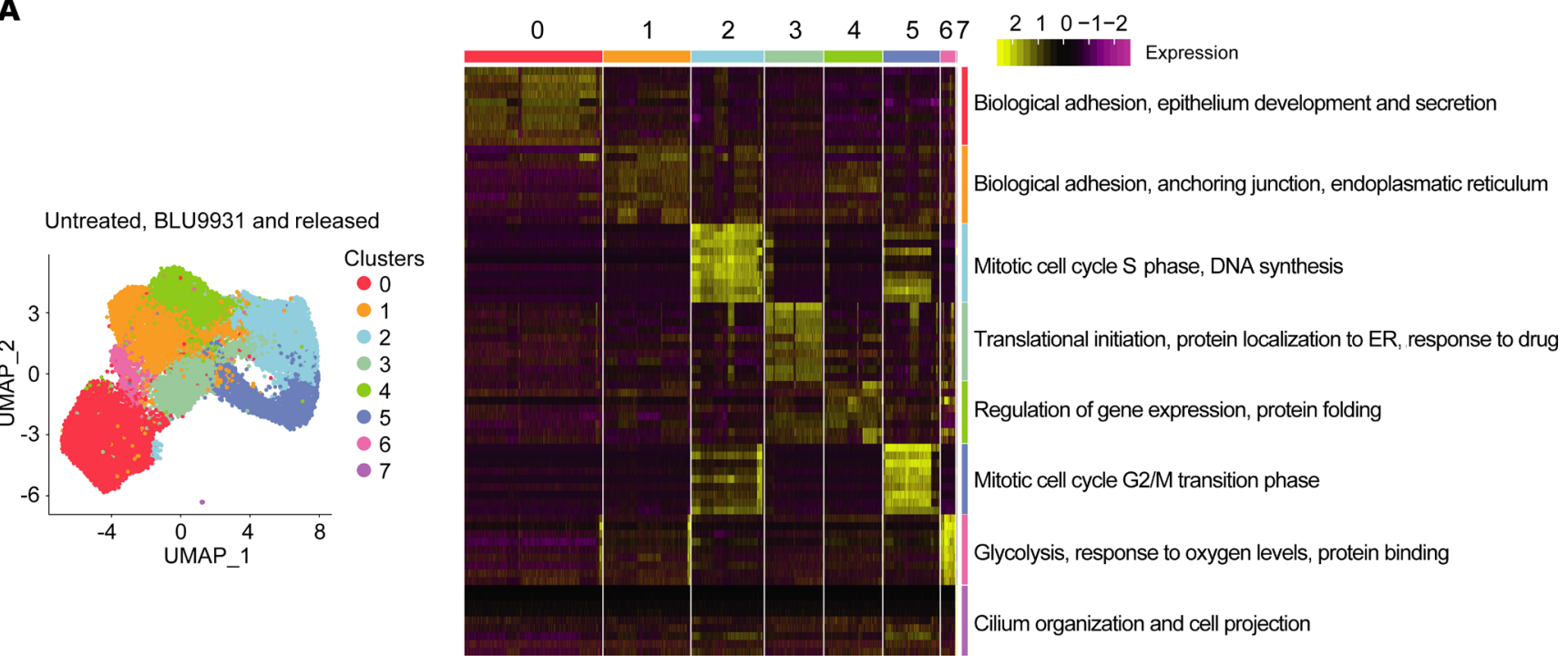

\section{B}

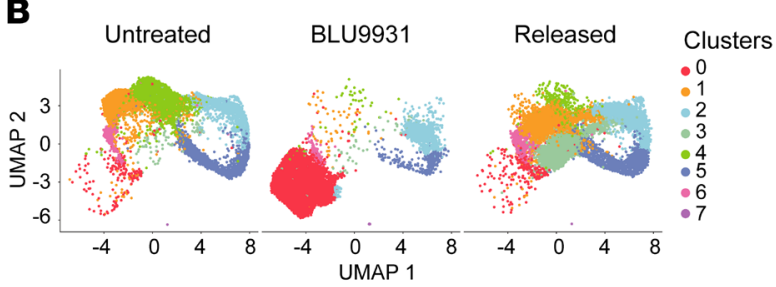

D

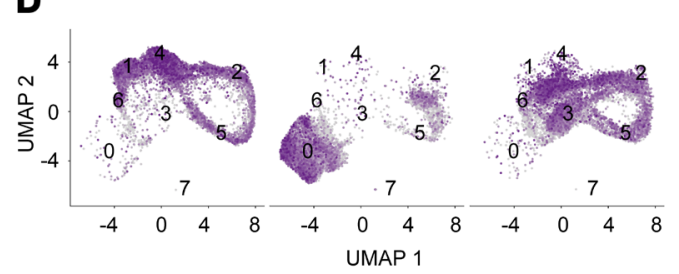

F

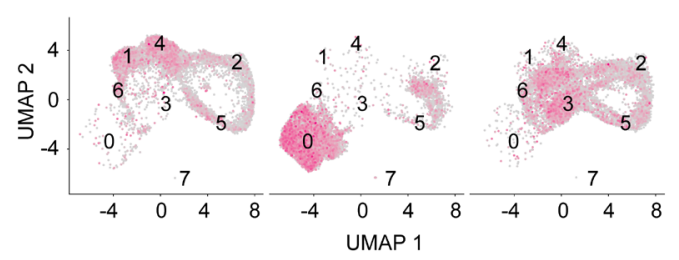

H

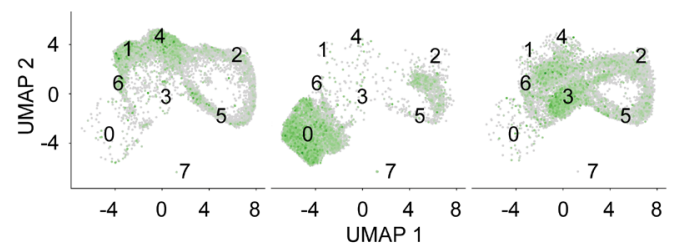

C

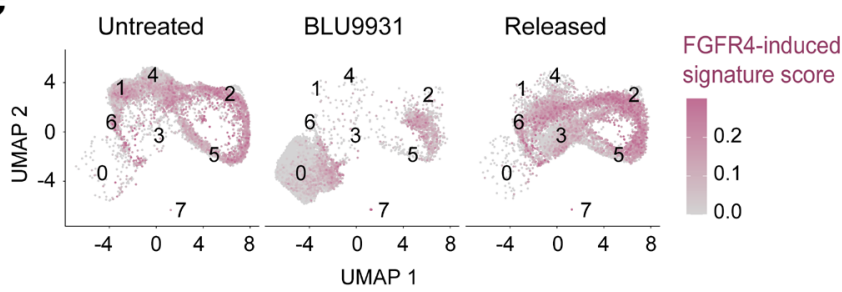

E

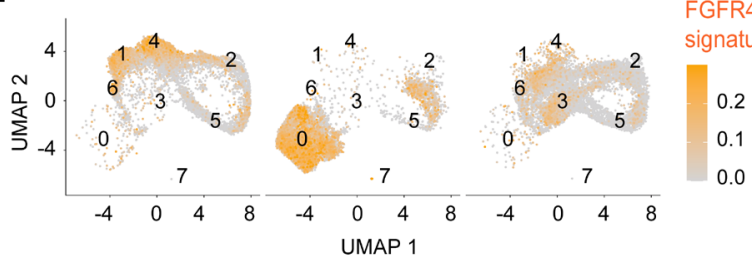

G

ERBB2

2.5
2.0

2.0
1.5

1.0

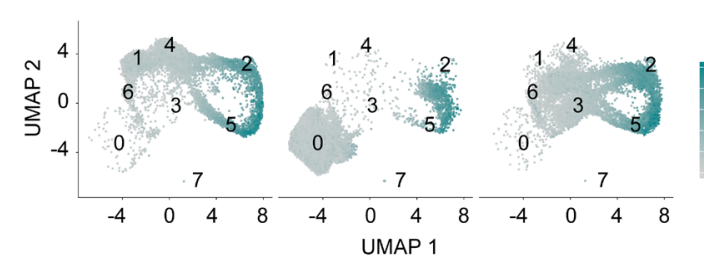

I

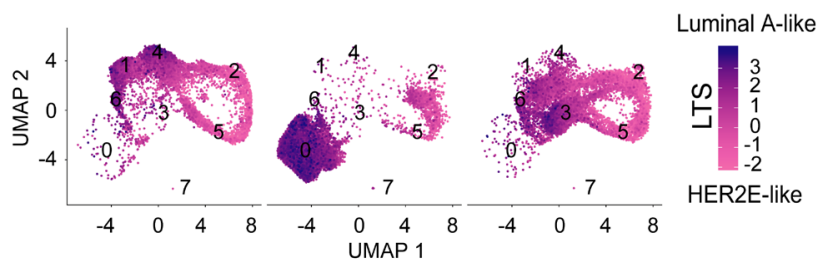


Figure 4. Single-cell RNA sequencing of WHIM11 tumor treated with BLU9931 and drug released. (A) Left panel: Uniform manifold approximation and projection (UMAP) plot of all combined cells (30,058 cells in total) that passed quality checks in untreated WHIM11 ( $n=2 ; 9298$ cells), treated with BLU9931 ( $n=2 ; 9777$ cells) $(0.6 \mathrm{~g} / \mathrm{kg} /$ day) for 14 days, and treated but released of drug for 18 days ( $n=2 ; 10,983$ cells). Cells and clusters are color coded by each cell population found. Right panel: Heatmap of significantly differentially expressed genes and main Gene Ontology annotations for each cluster in WHIM11 based on MSigDB. Significant genes were identified by Wilcoxon's signed-rank $t$ test. (B) UMAP plot of all the cells that passed quality checks in WHIM11 and divided by each experimental condition as untreated, treated with BLU9931 ( $0.6 \mathrm{~g} / \mathrm{kg} /$ day) for 14 days, and treated but released of drug for 18 days. Cells and clusters are color coded by each cell population found. (D, F, and $\mathbf{H}$ ) UMAP plots showing the expression of FGFR4, ERBB2, and ESR1 genes across all WHIM11 clusters in WHIM11 untreated, treated with BLU9931 (0.6 g/ $/ \mathrm{kg} /$ day) for 14 days, and treated but released of drug for 18 days. (C, E, G, and I) UMAP plots showing the gene signature score (average values of genes present in each score respectively for each cell) of FGFR4-induced signature, FGFR4-repressed signature, proliferation signature, and luminal tumor score (LTS) across all WHIM11 clusters in WHIM11 untreated, treated with BLU9931 $(0.6 \mathrm{~g} / \mathrm{kg} /$ day) for 14 days, and treated but released of drug for 18 days.

6B). Thus, from a DNA marker perspective, BLU9931 treatment did not alter tumor clonality.

Next, we performed scRNAseq analysis on the untreated, 14-day BLU9931-treated, and 14-day BLU9931-treated and drug-released WHIM11 tumors to further examine intratumor heterogeneity. Analysis of resulting transcriptomic data using the Seurat package (https://satijalab.org/seurat/) identified 8 different cell subpopulations in WHIM11 tumors (Figure 4A). Major biological terms enriched in these groups were identified using MSigDB (refs. 43, 44 and Supplemental Table 8). FGFR4, ERBB2, and ESR1 genes were expressed in all cell clusters. Cluster 0 was enriched for luminal-like features including cell differentiation and cell-cell adhesion terms, and showed high expression of ERBB2 and ESR1 (Figure 4, B, F, and H, and Supplemental Figure 8, B and C). In addition, cluster 0 had lower expression of FGFR4, and was mainly present in the BLU9931-treated group (Figure 4, B-D, and Supplemental Figure 8A). Moreover, clusters 3 and 6 were mainly seen after drug release. These clusters were related to translational initiation and glycolysis, respectively, probably in response to nutrient availability and mitogenic stimulation after drug release (Figure 4A and Supplemental Table 8). Clusters 2 and 5 were enriched in $\mathrm{G} 2 / \mathrm{M}$ phase- or S phasespecific genes (45), respectively (Figure 4A, and Supplemental Table 8). Clusters 1 and 4 were similar and enriched in the untreated and released experimental groups and had higher FGFR4 gene expression values linked to cell adhesion and regulation of gene expression, respectively (Figure 4, A and D, and Supplemental Table 8). Interestingly, cluster 7 was formed by a small number of cells ( 25 cells) and enriched in treated samples only; the gene expression pattern of cluster 7 was very specific and related to cilium organization and cell projection (see genes in Supplemental Table 8). Depletion of proliferation-related genes in treated tumors like NEK2 and AURKA, as demonstrated through proteomic profiling (Supplemental Figure $4 \mathrm{~B}$ ), were closely related to cilia formation in breast cancer $(46,47)$ and growth factors like FGF contribute to their resorption (48). Based on the lower number of cells present in cluster 7 compared with the rest of the clusters (Supplemental Table
8), we did not consider this cluster for further expression score quantification. By comparing all cells of each experimental group, the average expression of FGFR4 was significantly lower, and that of ERBB2 and ESR1 significantly higher, for the BLU9931-treated group, supporting the bulk-tumor gene expression arrays (Supplemental Figure 7H).

We also employed the previously developed FGFR4 signatures and the LTS in the scRNAseq data. The FGFR4-induced signature was lower in cluster 0 but higher in clusters 1,2 , and 5 (Figure 4C and Supplemental Figure 8D). FGFR4-repressed signature was higher in cluster 0 (Figure 4E and Supplemental Figure $8 \mathrm{E}$ ). Proliferation signature score showed that highly proliferative cells decreased after BLU9931 treatment (clusters 2 and 5) (Figure $4 \mathrm{G}$ and Supplemental Figure $8 \mathrm{~F}$ ), and rebounded upon drug removal. The LTS showed that cluster 0 was the most luminal A-like cell population, while clusters 2 and 5 were the most HER2E-like (Figure 4I and Supplemental Figure 8G). Clusters 1 and 4 were a mixture of FGFR4-induced and -repressed signatures as well as HER2E and luminal A phenotypes (Figure 4, C, E, and I, and Supplemental Figure 8, D, E, and G). The average expression of the signatures depicted in Figure 4, C, E, G, and I, and grouped by the 3 experimental groups was significantly lower for FGFR4-induced and proliferation signatures and significantly higher for the FGFR4-repressed signature and LTS in the BLU9931-treated group (Supplemental Figure 8I). Together, these data suggest that BLU9931 treatment does not have selective activity on specific subclones within WHIM11 tumors, but rather acts on all cells, with alterations in expression profiles supporting dynamics in cellular plasticity.

FGFR4-derived gene signatures are associated with multiple clinical features. To further understand the FGFR4-associated in vivo phenotypes in breast cancer patients, both FGFR4 signatures were used to determine a gene signature score calculated as the mean expression value for all genes in a signature, calculated for each patient in TCGA data set. This analysis showed that the FGFR4induced signature was highly expressed in the HER2E-subtype tumors, but also in basal-like tumors (Figure 5A). Conversely, the FGFR4-repressed signature was enriched in luminal A tumors (Figure 5A). In addition, we included histologic subtypes (i.e., invasive lobular carcinoma [ILC], invasive ductal carcinoma [IDC], and mixed [ILC+IDC]) in the molecular subtyping classification (49) as a separate group, where the FGFR4-repressed signature was high in ILC tumors (Supplemental Figure 3C).

To further test if these 2 FGFR4 signatures are robust discriminators of tumor differentiation, we calculated the correlation between the 2 FGFR4 signatures and the LTS using TCGA data set. The FGFR4-induced signature negatively correlated with LTS (Pearson's $r=-0.4588, P=2.082 \times 10^{-63}$ ) (Figure 5B), while the FGFR4-repressed signature showed a positive correlation with the LTS (Pearson's $r=0.8318, P=5.717 \times 10^{-308}$ ) (Figure $5 B)$. To further test if either FGFR4 signature was associated with the pathological measure of tumor differentiation (i.e., grade), we tested the association of FGFR4 signatures with tumor grade using the METABRIC cohort (1671 patients). As shown in Figure $5 \mathrm{C}$, the FGFR4-induced signature was associated with high grade (Figure 5C), while the FGFR4-repressed signature was associated with low grade (Figure $5 \mathrm{C}$ ). 
A
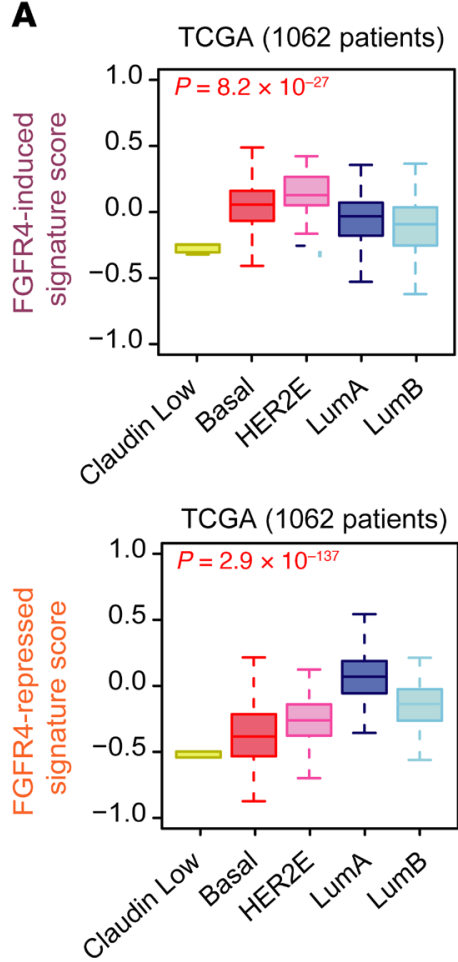

B
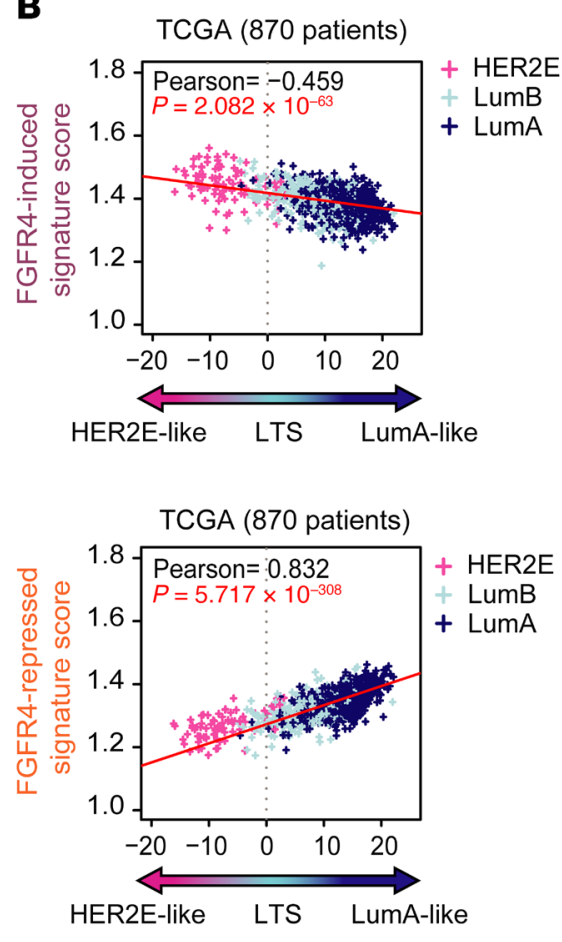

C
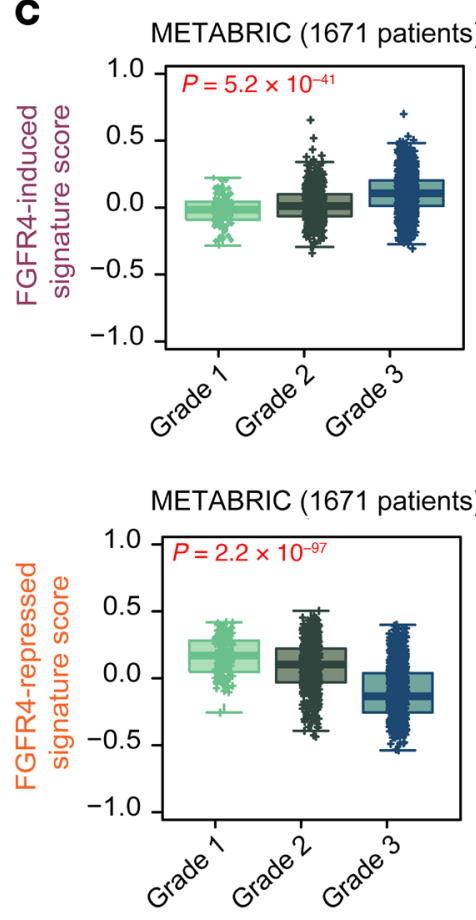

D

FGFR4-induced signature METABRIC 1672 patients

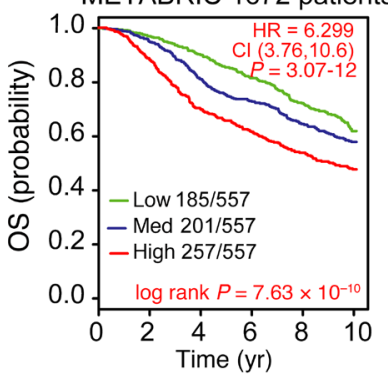

E

FGFR4-induced signature MDACC 446 patients

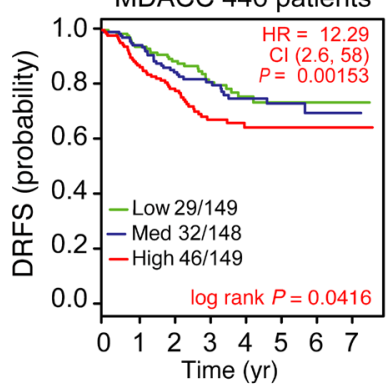

FGFR4-repressed signature METABRIC 1672 patients

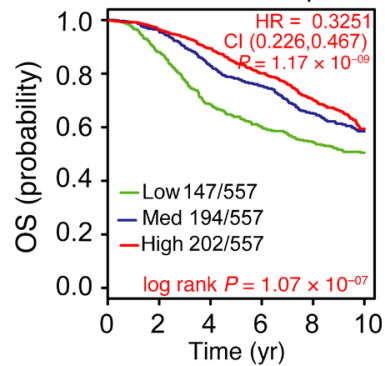

FGFR4-repressed signature

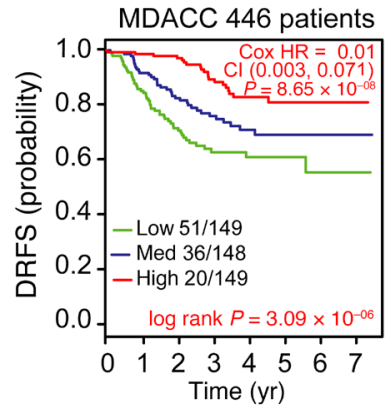

$\mathbf{F}$

METABRIC 1671 patients

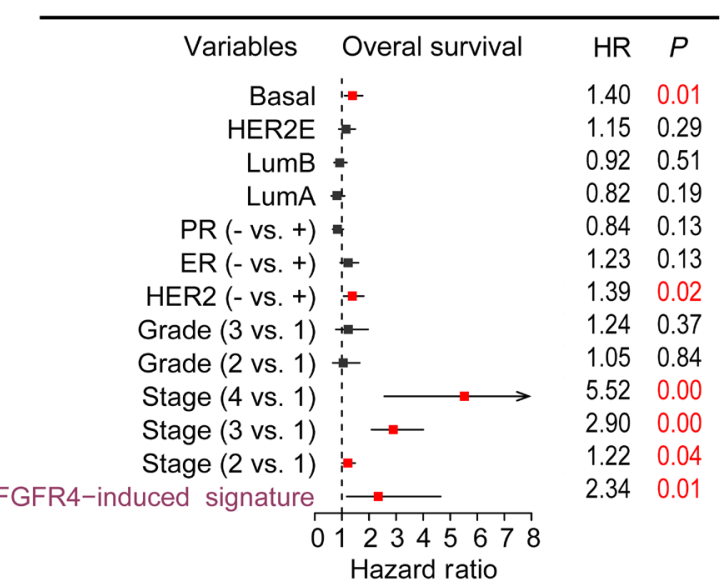


Figure 5. Prognostic value of FGFR4-derived signatures. (A) Average expression of FGFR4-related signatures in TCGA tumor molecular subtypes. Normal-like patients and true-normal tissues have been removed from the analysis. Statistical differences were calculated by ANOVA test. (B) Scatterplot showing the correlation between FGFR4-related signatures and luminal tumor score (LTS) (as calculated in TCGA data set using only HER2E, luminal A, and luminal B tumors). Correlation was measured using the Pearson correlation coefficient. (C) Average expression of FGFR4related signatures depending on histological tumor grade in METABRIC data (Grade 1: Low grade or well differentiated; Grade 2: Intermediate grade or moderately differentiated; Grade 3: High grade or poorly differentiated). ( $\mathbf{D}$ and $\mathbf{E}$ ) Kaplan-Meier plots to test the prognostic ability of FGFR4 signatures in METABRIC (D) and MDACC (E) data sets (normal-like samples were removed from the analysis in both cohorts). Survival curve differences were calculated by the log-rank test and the estimates of survival probabilities and cumulative hazard with a univariate Cox proportional hazards model. (F) Multivariable Cox proportional hazards analyses using METABRIC data (normal-like samples were removed from the analysis). Hazard ratio $(H R)=$ 1: no effect. $H R<1$ : reduction in hazard. $H R>1$ : increase in hazard. Signatures were evaluated as continuous variables and rank ordered according to the gene FGFR4 signature scores (induced and repressed) in 3 different levels: low, medium, and high (assigned by distribution in a given upper, middle, or lower tertile). Comparison between more than 2 groups was performed by ANOVA. Statistically significant values are highlighted in red. Box-and-whisker plots display the median value on each bar, showing the lower and upper quartile range of the data and data outliers. The whiskers represent the interquartile range. Each mark represents the value of a single sample. LumA, luminal A; LumB, luminal B.

Next, we tested the prognostic ability of the FGFR4 signatures in the METABRIC data set (Figure 5D) and MDACC breast cancer data set (477 patients) (Figure 5E). For this analysis, we rank ordered the patients into tertiles according to their FGFR4induced and FGFR4-repressed signature scores. As demonstrated by Kaplan-Meier analysis, both FGFR4 signatures were significantly associated with overall survival (OS). Patients with a high FGFR4-induced signature had a lower OS rate $(\mathrm{HR}=6.299, P=$ $3.07 \times 10^{-12}, \mathrm{CI}=3.76-10.6$ ) (Figure 5D) and patients with a high FGFR4-repressed signature presented better prognosis $(\mathrm{HR}=$ $0.3251, P=1.17 \times 10^{-9}, \mathrm{CI}=0.226-0.467$ ) (Figure 5D). Additionally, high scores of the FGFR4-repressed signature were strongly associated with a lower distant recurrence-free survival (DRFS) (HR $=0.01668, P=7.32 \times 10^{-8}, \mathrm{CI}=0.00376-0.074$ ) (Figure $5 \mathrm{E}$ ) and FGFR4-induced signature with a higher DRFS (HR $=13.39, P=1.42$ $\times 10^{-3}, \mathrm{CI}=2.72-66$ ) (Figure $5 \mathrm{E}$ ). A multivariate analysis using the METABRIC data set and the covariates of PAM50 subtypes, ER and PR status, and histological grade and tumor stage showed that the FGFR4-induced signature $\left(\mathrm{HR}=2.41, P=1.0 \times 10^{-2}, \mathrm{CI}=0.01-4.65\right)$ was a significant independent prognostic factor (Figure $5 \mathrm{~F}$ ).

Transcriptome comparison between primary and paired metastatic tumors. Previous work by our group using a panel of 105 genes showed that FGFR4 was one of the top genes highly expressed in metastasis compared with the matched primary tumors (4). To further define a possible role for FGFR4 in metastasis, we assayed 103 primary tumor-metastasis pairs using RNAseq and tested our FGFR4 signatures alongside hundreds of genomic signatures for differential expression across this breast tumor metastatic landscape. All tumors were assigned to an intrinsic molecular subtype of breast cancer using the previously reported (4) PAM50 subtype predictor (except 1 primary paired metastases with missing sub- type information) (Supplemental Table 1). We first determined the distribution of the PAM50-intrinsic subtypes across this cohort (Supplemental Figure 9E and Supplemental Table 1). This allowed us to avoid the possible confounding factor of intrinsic subtype in the subsequent analysis, as we divided tumors into 2 data sets based on the subtype of the primary tumor from each pair: a "luminal set" comprising all luminal A/B- and HER2E-subtype patients (77 pairs) (Supplemental Figure 9A) and a "basal-like set" containing 8 basal-like-only pairs (Supplemental Figure 9B); samples (and the pair) called normal-like in either the primary or metastatic tumors were removed from the analysis (Supplemental Figure 9, A, B, and D). The subtype discordance rate between primary and metastatic tumors in the luminal data set (Supplemental Figure 9A) and the entire data set (Supplemental Figure 9C) showed that luminal A tumors had the highest discordance rate and often changed to luminal B or HER2E (37.5\% and 15\%, respectively), which is in agreement with previous results (4). The basal-like subtype was maintained in all samples as basal-like (100\%) (Supplemental Figure 9B), and only 2 HER2E primary tumors were called basal-like in the metastases (16.6\%); the sites and number of metastases are summarized in Supplemental Figure $9 \mathrm{~F}$ and Supplemental Table 1.

We next performed a supervised 2-class paired analysis to identify genes significantly upregulated in metastasis. From luminal A/B and HER2E tumors (luminal set), we detected 119 upregulated and 487 downregulated genes in metastatic tumors at an FDR of O. FGFR4 was the only FGFR family member significantly upregulated and was among the highest differentially expressed genes. Analyzing our primary and metastatic paired samples, $13 \%$ of all primary tumors had high expression of FGFR4 considering all subtypes, and $60 \%$ of all HER2E subtype tumors had high expression of FGFR4 by mRNA (FGFR4 mRNA expression was considered high expression when it was greater than $75 \%$ of positive values in RNAseq median-centered data) (Supplemental Figure 9G).

Other key genes expressed in luminal tumors and typically associated with a better prognosis including PGR, ESR1, BCL2, SLC39A6, GATA3 and NAT1 were downregulated in luminal tumor metastases, suggesting that the majority of the metastatic tumors were losing luminal properties. Genes significantly higher in luminal metastases beyond FGFR4 included PTTG1, BIRC5, CCNB1, CDC6, and AURKA; these genes are associated with proliferation and cell cycle processes (Supplemental Table 9), and were highly expressed in luminal B and HER2E compared with luminal A tumors. Genes related to the HER2E subtype (35, 50-53), neutrophils, monocytes, and myeloid-derived suppressor cells (MDSCs) (54-56) such as S100A9, S100A8, and others like TDO2 $(57,58)$, were also upregulated in metastases compared with primary tumors (Supplemental Table 9).

Basal-like tumors showed many fewer differences in gene expression than luminal paired tumors, resulting in only 5 genes (RN7SL314P, ZBTB8B, PKMP3, NYAP1, and METTL21EP) demonstrating significant changes (Supplemental Table 9). To test whether differentiation states are altered between primary tumors and paired metastases, we calculated the LTS in the luminal paired tumors and determined that it was significantly lower in metastases compared with the primary tumors (Figure 6A). To test the association of FGFR4 activity with this process, we next tested 
A

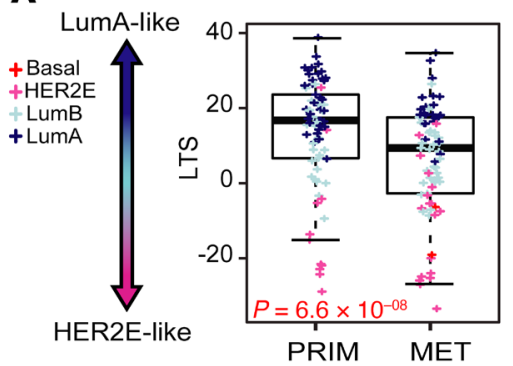

B

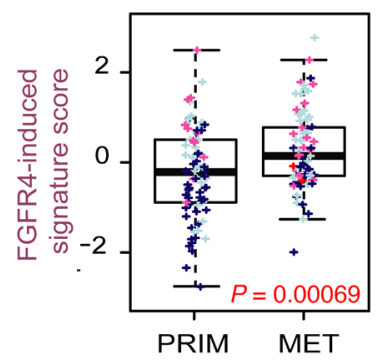

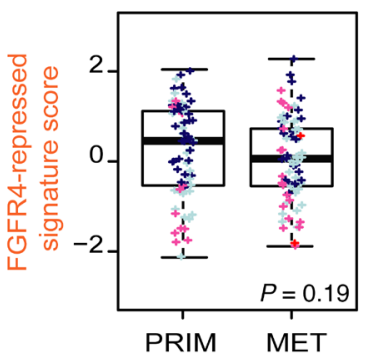

C

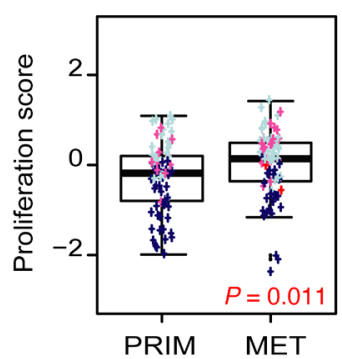

D

Luminal set

77 Primary and metastatic pairs (154 tumors)

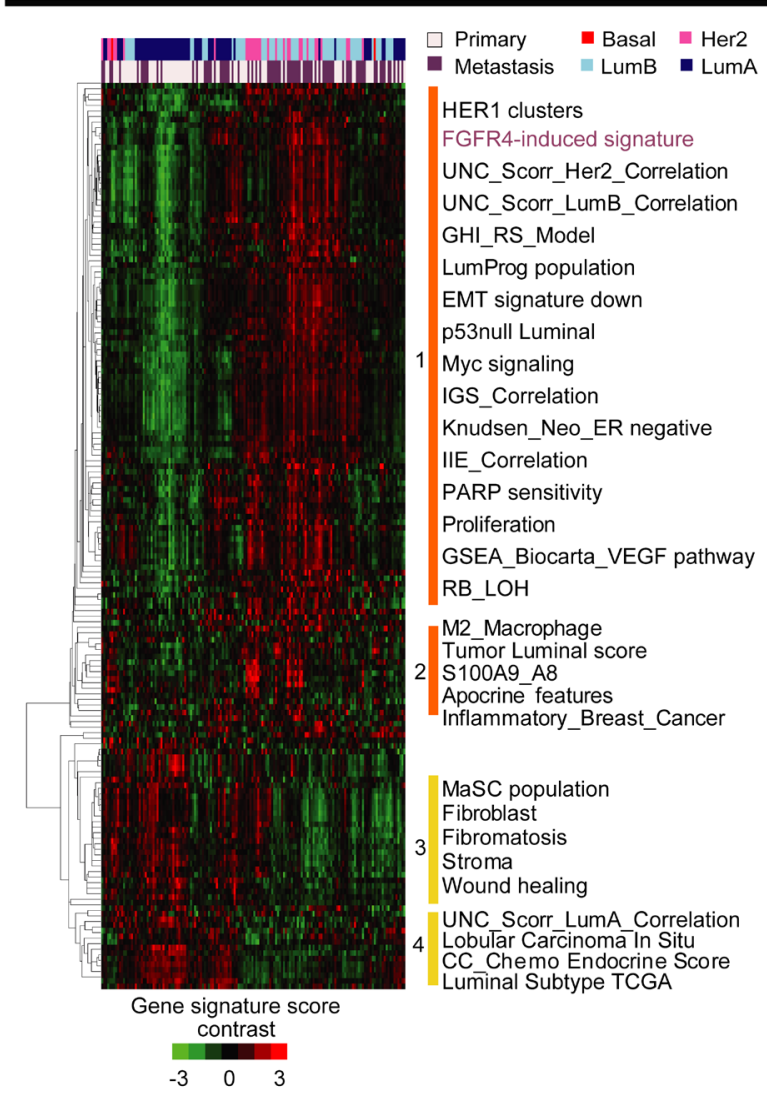

E

Basal set

8 Primary and metastatic pairs (16 tumors)

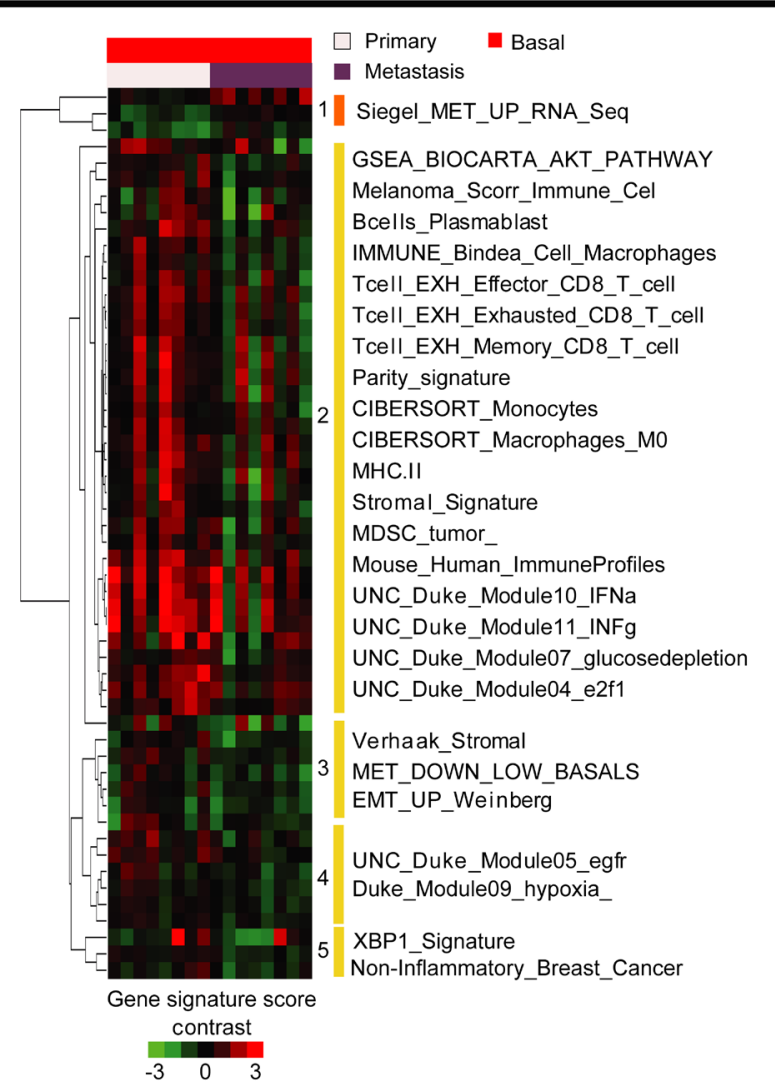

Figure 6. Expression profile and FGFR4-associated signatures in matched breast primary tumors and metastases. (A) Luminal tumor score (LTS) calculated values in 77 primary and 77 paired metastatic tumors in the luminal set. (B) Expression levels of FGFR4-induced (left) and FGFR4-repressed signatures (right) in 77 primary and 77 paired metastatic luminal tumors. (C) Proliferation scores for FGFR4-induced and -repressed signatures (see PAM50 subtype classification in Methods) in 77 primary and 77 paired metastatic luminal tumors. (D) Supervised hierarchical cluster analysis derived from the significantly different modules scores, in luminal and (E) basal sets. Significance of the differences between modules was calculated using 2-class SAM at an FDR of $0 \%$. Significantly up- and downregulated modules are clustered together for each set (basal and luminal). Clusters ( 4 from luminal and 5 from basal set) were selected based on node correlation greater than 0.5 . Comparisons between 2 paired groups were performed by paired, 2 -tailed $t$ test. Statistically significant values are highlighted in red. Box-and-whisker plots display the median value on each bar, showing the lower and upper quartile range of the data and data outliers. The whiskers represent the interquartile range. Each mark represents the value of a single sample. LumA, luminal A; LumB, Iuminal B; PRIM, primary tumor; MET, metastatic tumor.

the newly developed FGFR4 signatures and proliferation scores in tumor-met pairs. Here, we found the FGFR4-induced signature and proliferation score were significantly higher in metastases compared with the primary tumors (Figure 6, B and C). Likewise, metastatic tumors trended toward a lower FGFR4-repressed signature score (Figure 6B). No significant result was found in basallike paired samples for either FGFR4 signature or proliferation
(Supplemental Figure 10, A and B). Together, these data illustrate that some luminal primary tumors lose luminal-like properties and gain enhanced FGFR4 activity concomitant with HER2Esubtype features in metastases.

To understand what other pathways and processes associate with FGFR4 in established metastases, we analyzed 696 prespecified gene expression modules $(35,59)$ alongside 
A

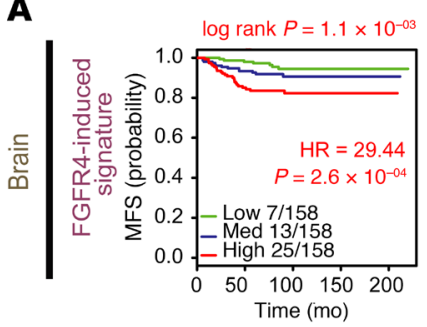

C

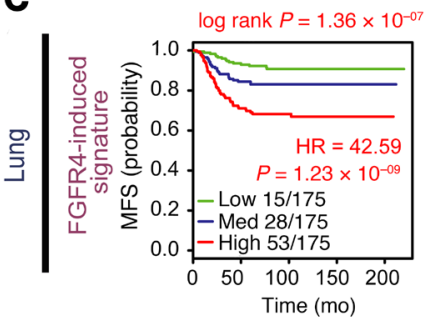

E

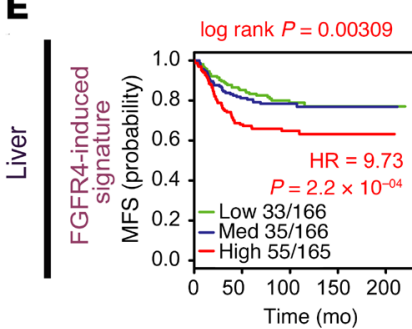

$\log \operatorname{rank} P=3.39 \times 10^{-05}$

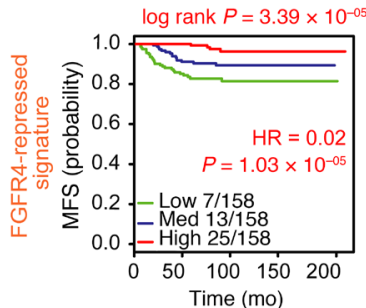

$\log$ rank $P=7.76 \times 10^{-08}$

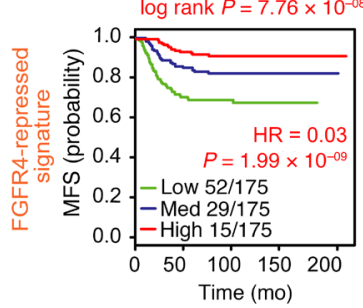

log rank $P=0.00617$

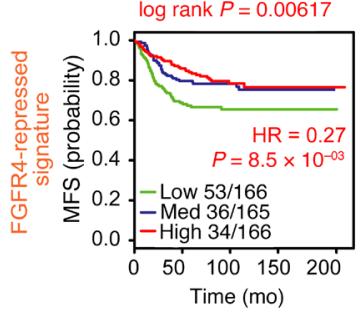

B

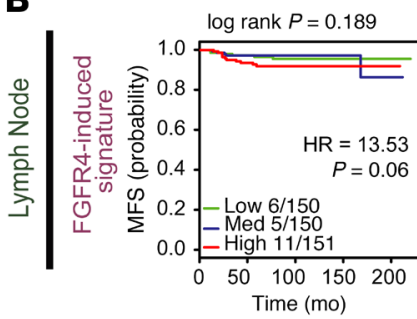

D

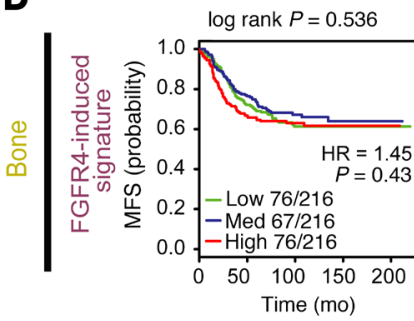

$\mathbf{F}$

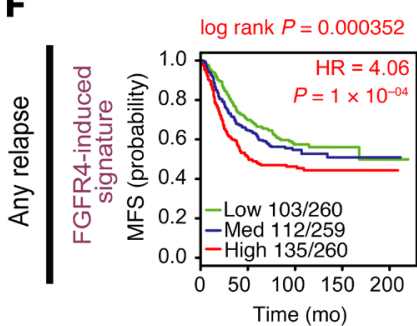

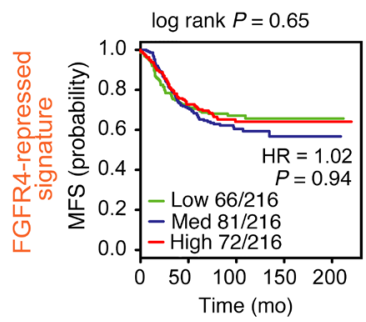
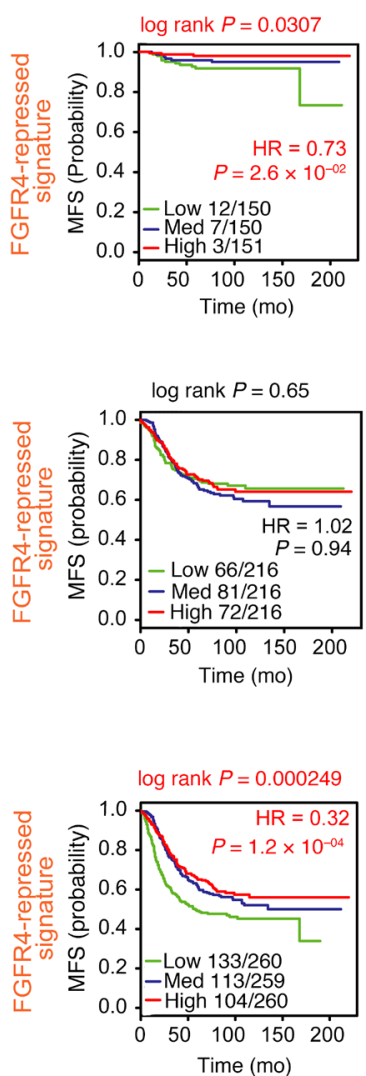

Figure 7. Univariate analysis in $\mathbf{8 5 5}$ primary tumors with known first site of relapse. Kaplan-Meier and Cox proportional hazards model analyses of metastasis-free survival (MFS) analyzed in their specific sites of relapse: brain (A), lymph node (B), lung (C), bone (D), liver (E), or any site of relapse (F). Survival curve differences were calculated by the log-rank test and the estimates of survival probabilities and cumulative hazards with a univariate Cox proportional hazards model. FGFR4-derived signatures were evaluated as continuous variables and rank ordered according to the gene FGFR4 signature scores (induced and repressed) in 3 different levels: low, medium, and high (assigned by distribution in a given upper, middle, or lower tertile). Hazard ratio $(H R)=1$ : no effect. $H R<1$ : reduction in the hazard. $H R>1$ : increase in hazard. Statistically significant values are highlighted in red.

the new FGFR4 signatures (Supplemental Table 10) in the 77 paired luminal and 8 paired basal-like tumors. Supervised analysis identified 119 upregulated modules and 43 downregulated in the luminal set (Supplemental Table 9). A supervised hierarchical cluster derived from the significantly differentially expressed modules showed 4 main clusters of signatures (Figure 6D). Clustering results revealed that certain primary tumors and metastases were more similar, but the dominant trend was distinction between metastases and primary tumors. As expected, the FGFR4-induced signature was significantly higher in metastases compared with primary tumors (cluster 1 ), as were proliferation signatures (Figure 6D). In addition, the FGFR4-induced signature demonstrated a strong relationship with high expression of signatures related to proliferation, angiogenesis, HER2E/luminal B features, luminal progenitor features, and downregulation of EMT. Interestingly, we also found tight covariance between M2 macrophage and inflammatory breast cancer signatures, and the newly defined LTS with other signatures closely related to the HER2E subtype. In fact, a marker panel specific to M1 (CD68) or M2 macrophages (MRC1, CD163, ARG1, MERTK, and MACRO) (60-62) showed significantly higher expression in luminal metastases, but not in basal-like metastatic tumors (Supplemental Fig- ure 11, A and B); this suggests that macrophage infiltration is associated with metastases in luminal/HER2 cancers (with most other immune features being unchanged). Conversely, modules downregulated in luminal metastasis were grouped in clusters 3 and 4, and composed mainly of stroma-related signatures, mammary stem cell (MaSC), and luminal subtype signatures (Figure 6D).

To further explore a possible relationship between FGFR4 expression and genetic alterations we used the RNAseq data to identify mutations in FGFR4. Other studies have estimated that FGFR4 is mutated in 1\%-3\% of cases, altered at a higher rate in the metastatic setting (63-65). Considering only the mutations found in COSMIC $(66,67)$, we analyzed the mutation calls on 206 samples for FGFR4 and identified 9 variants (5 primary and 4 metastatic unpaired tumors) as being mutated (4\% of cases) (Supplemental Table 11). Otherwise, if we consider mutations in COSMIC that were described only in breast cancer (63-65), a single primary tumor harbored an FGFR4 mutation (0.5\% mutated), demonstrating again that high expression of FGFR4 is probably nongenetically determined.

Supervised analysis of the 8 paired basal-like tumors, using an FDR of 0 , identified 3 upregulated and 51 downregulated modules in basal-like metastasis compared with the primary 
tumors (Supplemental Table 9). A second supervised cluster only derived from the significant modules showed 5 new clusters (Figure 6E). A signature recently published by our group as being more highly expressed in metastases derived from a different data set (68) was also significantly higher here, but only in basal-like metastases. Clusters 2, 3, 4, and 5 were modules downregulated in metastatic basal-like tumors and where cluster 2 presented a decrease in immune signatures related to effector $\mathrm{CD}^{+} \mathrm{T}$ cells and memory $\mathrm{CD}^{+} \mathrm{T}$ cells, B cells, monocytes, MO macrophages, IFN- $\gamma$ and IFN- $\alpha$ signaling, and MDSCs (Figure 6E, Supplemental Figure 10C, and Supplemental Table 9). No changes were detected for M2-like profiles or FGFR4 signatures. EMT, stromal, and the downregulated signature derived from the matched breast cancer primary tumors and multiorgan metastases by Siegel et al. (68) defined cluster 3 . These data suggest distinct changes in the metastatic setting when luminal/ER ${ }^{+}$ and basal-like tumors are compared.

FGFR4-associated signatures predict metastasis-free survival in visceral metastasis. We and others have previously shown that the PAM50 subtype predicts site of metastasis (69-71). Therefore, we sought to analyze the prognostic capacity of the 2 FGFR4 signatures in 855 primary tumors with documented first site of relapse (69). The FGFR4-induced and -repressed signatures were strongly associated with risk of metastasis using multiple sites of first relapse (Figure 7F). Kaplan-Meier and Cox proportional hazards model analyses detected strong significant associations of FGFR4-induced signature with high risk for metastasizing to the brain, lung, and liver (Figure 7, A, C, and E), but not to lymph nodes or bone (Figure 7, B and D). Likewise, high expression of the FGFR4-repressed signature predicted better prognosis in brain, lung, liver, and lymph node (Figure 7, A-C and E) but not in bone (Figure 7D).

Accounting for intrinsic subtype indicated that FGFR4related signatures predicted distant recurrence in any subtype of luminal tumors (luminal A, luminal B, and HER2E), but not in basal-like or claudin-low tumors (Supplemental Figure 12). Next, using a multivariate analysis combining known clinical risk factors and dividing the tumors in basal-like and luminal groups, FGFR4induced signature provided significant and independent prognostic information in only the luminal group for brain, liver, and lung metastasis (Supplemental Figure 13A). Finally, the FGFR4repressed signature predicted risk of distant recurrence only in luminal group tumors that relapse in brain (Supplemental Figure 13B). Precisely why the FGFR4-induced signature shows predilection for the brain, liver, and lung requires further investigation, but we hypothesize that there could be microenvironmental features (either growth factors or specific cell-cell contacts) that facilitate growth of FGFR4 $4^{+}$, HER2E tumors at these sites.

\section{Discussion}

The impact of genetics and genomics in breast cancer has improved our ability to efficiently predict the risk of recurrence and response to therapy, in a patient-specific and efficient manner. Here, we developed 2 robust FGFR4-associated signatures using a HER2E/cHER2 ${ }^{-} / \mathrm{ER}^{+}$PDX model to explore the biological significance of FGFR4 genomic signaling in breast cancer. The molecular profile obtained from this in vivo approach, the activation state of the kinome, and the scRNAseq data demonstrate that FGFR4 activity could be regulating tumor plasticity and actively repressing many genes involved in luminal tumor cell differentiation and inducing genes involved in the more proliferative and metastatic HER2-enriched phenotype. We demonstrated that in PDX WHIM11, FGFR4 is highly activated and triggers MAPK/ERK and PI3K cascades. How FGFR4 activation occurs in HER2E breast cancers is still unknown. There is evidence that FGFR4 is activated via binding to specific ligands, and can dimerize and activate in the absence of FGF ligand(s) through ligand-independent mechanisms (72-74); based on data mining of the genomics data, it is not obvious which means of activation is occurring. Although we hypothesize that FGFR4 drives subtype switching, further study is needed to gain more insight into what is triggering FGFR4 activation.

In vitro experiments overexpressing FGFR4 in 2 luminal cell lines, or blocking FGFR4 activity, corroborated that FGFR4 activity is associated with subtype switching. Our data also suggested that cells genetically modified to overexpress FGFR4 were more HER2E-like than their counterpart controls or those treated with an FGFR4 inhibitor. The FGFR4-induced signature was strongly prognostic and high expression predicts low OS/DRFS, even in a multivariate analysis adjusting for subtype and multiple clinical features. Conversely, the FGFR4-repressed signature predicted a better prognosis.

The 77 primary tumor-metastasis pairs analyzed confirmed that the major differences found between primary and metastatic tumors were found in the luminal subset. The highest subtype-change rate was found from luminal A toward luminal B or HER2E. The newly derived LTS supported this hypothesis, allowing us to rank the level of differentiation between the luminal subtypes (HER2E and luminal A/B) and providing an objective means of assessing genomic-based tumor differentiation status within these $\mathrm{ER}^{+}$/luminal tumors. Around $40 \%$ to $50 \%$ of patients with luminal breast cancer experience relapses that include distant metastases $(75,76)$. Therefore, since they represent the most common subtype of the disease, luminal tumors are responsible for most breast cancer deaths. In clinical practice, treatment decisions are mostly based on the primary tumor phenotype; however, if we do not take into consideration evolution between the primary and metastatic tumor as demonstrated here, we may be missing a number of key therapeutically relevant tumor targets, including FGFR4.

Interestingly, in our study those primary tumors showing high luminal-like features (high LTS), mostly luminal A tumors, had the lowest LTS (more HER2E-like) in the metastatic site, while the HER2E and luminal B primary tumors remained relatively unchanged. These results suggested that under the influence of the microenvironment, treatment selection pressure, and probably with the increase/selection of cells with a higher FGFR4 activity, the more luminal A-like tumors could be more susceptible to change their phenotype in the metastatic setting. FGFR4 activation may promote cell proliferation and dedifferentiation, and ultimately tumor subtype switching, becoming a driver of metastasis in these luminal tumors. We again note that this high FGFR4 expression is not genetically driven (neither amplified nor activating mutations), and thus this key drug target will be missed when using DNA-based tests. 
The analysis of 696 expression modules revealed that besides proliferation and FGFR4 signatures, luminal-like metastases were associated with HER2E phenotype and luminal progenitor features, and only a few modules related to immune signatures including an M2 macrophage signature were more highly expressed in metastases.

Conversely, many fewer significant changes were found in basal-like metastatic tumors relative to primary, with the major changes being decreases in the vast majority of immune signatures (B cells and T cells), signatures related to EMT, and stromal features, in agreement with previous studies (77-81). We did not observe any significant differences between primary and metastases in all proliferation-related scores or modules analyzed in basal-like paired tumors (Figure 6E, Supplemental Table 9, and Supplemental Figure 10B), contrasting with the luminal set (Figure 6, $\mathrm{C}$ and $\mathrm{D})$. Thus, there are distinct changes in the immune microenvironment according to tumor subtype, where in luminal-like tumors there is an increase in immunosuppressive macrophages, while in basal-like cancers there is a general downregulation of all immune cell processes.

Our findings also add context to prior studies where FGFR4 plays a role in the biological response to endocrine therapies in $\mathrm{ER}^{+}$breast tumors (82) and is enriched in endocrine-resistant ILC (41). In our study, we demonstrate the association between FGFR4 and the loss of luminal features, including ESR1 itself. Our established luminal cell lines overexpressing FGFR4 have lower ESR1 and LTS than controls cells. Similarly, in WHIM11 PDX tumors, FGFR4-targeted inhibition resulted in higher ESR1 expression and ESR1-related signatures. In the clinical setting, luminal metastases also have lower levels of ESR1, PGR, and luminal-related signatures compared with their primary tumor counterparts. Together, these data led us to test FGFR4's possible regulation of tumor growth independently of ESR1 and we found that overexpression of FGFR4 enables estrogen-independent growth (Supplemental Figure 6E). Thus, the increase in FGFR4 seen in our metastatic patients could be acquired as a long-term signaling adaptation. It becomes clear that patients analyzed in Levine and Meijer's studies with higher FGFR4 mRNA levels may predict poor clinical benefit of endocrine therapy $(41,82)$. In fact, there are several ongoing clinical trials using new pan-FGFR inhibitors, mostly in breast cancer patients with $\mathrm{ER}^{+} / \mathrm{HER} 2^{-} /$FGFR1-3 amplification (NCT03238196, NCT03344536) (83); the drugs used in these trials also impact FGFR4, and given that FGFR4 is rarely amplified or mutated, the FGFR4 $4^{+}$(by mRNA and/or protein) patients are not being included. In clinical practice, both cHER2 ${ }^{-}$/HER2E and cHER2 ${ }^{+}$/HER2E breast cancers are likely to be FGFR4 ${ }^{+}$, and thus may benefit from FGFR4 inhibitors. We suggest that high FGFR4 expression (by mRNA, protein, or by FGFR4-induced signature) could be considered as a possible inclusion criterion for patients to receive drugs targeting FGFR4. Last, we note that FGFR4 is not only highly expressed in cHER2 $^{-}$/HER2E tumors but also in cHER2 $^{+} /$HER2E and some basal-like tumors; thus, FGFR4 might also be a viable target in some basal-like tumors.

Finally, we demonstrated that these FGFR4-related signatures predicted not only survival, but also site-specific metastasis. We found strongest associations of FGFR4-induced signature with high risk of metastasizing to brain, lung, or liver but not to bone or lymph node. In agreement with an FGFR4-brain metastasis association, a genomic study of 20 paired primary and resected brain metastases also found the FGFR4 gene upregulated in $30 \%$ of brain metastases and ESR1 as the most recurrently downregulated gene (5). Thus, our study and those of others collectively suggest that the nongenetically defined FGFR4 signaling pathway plays an important role in the progression of luminal disease, predicts worse survival, and site-specific metastasis. Indeed, we show that many of these features can be disrupted using small molecules inhibiting FGFR4, for example, as we show using BLU9931. Thus, FGFR4 may be an attractive therapeutic target that could impact the metastatic setting and resistance to endocrine therapy.

\section{Methods}

Experimental details can be found in the Supplemental Methods.

Study approval. All human samples used here were either obtained from the public domain or were anonymized and deidentified (i.e., 103 primary tumors and associated metastases); these latter samples were ruled as Not Human Subjects Research.

\section{Author contributions}

SGR, CMP, and AP conceptualized the study. SGR, CMP, JSP, AT, JPG, MPE, and CF designed the study methodology. SGR, AT, SRS, $\mathrm{ARC}$, and DM interpreted the data and/or performed statistical analysis. SGR, AT, MPE, XH, KRM, JPG, and PG conducted experiments. SGR, CMP, AP, JSP, DPH, FBM, JMR, and LAC interpreted data and/or wrote the manuscript. CMP, AP, SGR, JMC, OB, JA, FR, AL, EMD, JMR, and GLJ were responsible for data curation and supervised the project. CMP and LAC provided resources. CMP supervised the study.

\section{Acknowledgments}

This work was supported by funds from the National Cancer Institute (NCI) Breast SPORE program (P50-CA58223), NCI grants R01-CA148761, R01-CA195740, and F32-CA210427 (to DPH); by the Breast Cancer Research Foundation; and by the Susan G. Komen grants SAC-160074 (to CMP) and PDF17479425 (to SGR). FBM was supported by the Fundación Científica Asociación Española Contra el Cáncer (AECC-Postdoctoral 17-1062). This work was also supported by Instituto de Salud Carlos III (PI16/00904 and PI19/01846), AACR Career Development Awards for Translational Breast Cancer Research, the Breast Cancer Research Foundation (19-20-26-PRAT), Breast Cancer Now (2018NovPCC1294), European Union's Horizon 2020 and innovation programme (H2020-SC1-BHC-2018-2020), Fundació La Marató TV3 (201935-30), Fundación Mutua Madrileña, and Fundación Quirón-Salud (all to AP). The results published here are in part based on data from TCGA managed by the NCI and the National Human Genome Research Institute (dbGaP accession phs000178). The authors thank the infrastructure and direct technical support received from High-Throughput Genomic Sequencing Facility (https://www.med.unc.edu/ genomics), Lineberger Animal Studies Core Facility (http:// unclineberger.org/research/core-facilities/research/corefacilities/animal-studies), Translational Pathology Lab (https:// 
www.med.unc.edu/pathology/tpl), Translational Genomics Lab (https://unclineberger.org/tgl), and Flow Cytometry Core Facility (https://www.med.unc.edu/flowcytometry) at the University of North Carolina at Chapel Hill.
Address correspondence to: Charles M. Perou, Lineberger Comprehensive Cancer Center, University of North Carolina at Chapel Hill, Chapel Hill, North Carolina 27599, USA. Phone: 919.843.5740; Email: cperou@med.unc.edu.
1. de Dueñas EM, et al. Prospective evaluation of the conversion rate in the receptor status between primary breast cancer and metastasis: results from the GEICAM 2009-03 ConvertHER study. Breast Cancer Res Treat. 2014;143(3):507-515.

2. Perou CM, et al. Molecular portraits of human breast tumours. Nature. 2000;406(6797):747-752.

3. Sørlie T, et al. Gene expression patterns of breast carcinomas distinguish tumor subclasses with clinical implications. Proc Natl Acad Sci US A. 2001;98(19):10869-10874.

4. Cejalvo JM, et al. Intrinsic subtypes and gene expression profiles in primary and metastatic breast cancer. Cancer Res. 2017;77(9):2213-2221.

5. Priedigkeit $\mathrm{N}$, et al. Intrinsic subtype switching and acquired ERBB2/HER2 amplifications and mutations in breast cancer brain metastases. JAMA Oncol. 2017;3(5):666-671.

6. Arteaga CL, Sliwkowski MX, Osborne CK, Perez EA, Puglisi F, Gianni L. Treatment of HER2positive breast cancer: current status and future perspectives. Nat Rev Clin Oncol. 2011;9(1):16-32.

7. Slamon D, et al. Adjuvant trastuzumab in HER2-positive breast cancer. $N$ Engl J Med. 2011;365(14):1273-1283.

8. Prat A, et al. Research-based PAM50 subtype predictor identifies higher responses and improved survival outcomes in HER2-positive breast cancer in the NOAH study. Clin Cancer Res. 2014;20(2):511-521.

9. Basho RK, McArthur HL. Optimizing (neo)adjuvant treatment of HER2-positive breast cancer. Ther Adv Med Oncol. 2018;10:1758835918775697.

10. Turner N, Grose R. Fibroblast growth factor signalling: from development to cancer. Nat Rev Cancer. 2010;10(2):116-129.

11. Beenken A, Mohammadi M. The FGF family: biology, pathophysiology and therapy. Nat Rev Drug Discov. 2009;8(3):235-253.

12. Ho HK, et al. Fibroblast growth factor receptor 4 regulates proliferation, anti-apoptosis and alpha-fetoprotein secretion during hepatocellular carcinoma progression and represents a potential target for therapeutic intervention.J Hepatol. 2009;50(1):118-127.

13. French DM, et al. Targeting FGFR4 inhibits hepatocellular carcinoma in preclinical mouse models. PLoS One. 2012;7(5):e36713.

14. Shah RN, Ibbitt JC, Alitalo K, Hurst HC. FGFR4 overexpression in pancreatic cancer is mediated by an intronic enhancer activated by HNF1alpha. Oncogene. 2002;21(54):8251-8261.

15. Zaid TM, et al. Identification of FGFR4 as a potential therapeutic target for advanced-stage, high-grade serous ovarian cancer. Clin Cancer Res. 2013;19(4):809-820.

16. Streit S, Bange J, Fichtner A, Ihrler S, Issing W, Ullrich A. Involvement of the FGFR4 Arg388 allele in head and neck squamous cell carcinoma. Int J Cancer. 2004;111(2):213-217.

17. Spinola M, et al. FGFR4 Gly388Arg polymor- phism and prognosis of breast and colorectal cancer. Oncol Rep. 2005;14(2):415-419.

18. Wang J, Stockton DW, Ittmann M. The fibroblast growth factor receptor-4 Arg388 allele is associated with prostate cancer initiation and progression. Clin Cancer Res. 2004;10(18 pt 1):6169-6178.

19. Bange J, et al. Cancer progression and tumor cell motility are associated with the FGFR4 Arg(388) allele. Cancer Res. 2002;62(3):840-847.

20. Huang KL, et al. Proteogenomic integration reveals therapeutic targets in breast cancer xenografts. Nat Commun. 2017;8:14864.

21. Li S, et al. Endocrine-therapy-resistant ESR1 variants revealed by genomic characterization of breast-cancer-derived xenografts. Cell Rep. 2013;4(6):1116-1130.

22. Hagel M, et al. First selective small molecule inhibitor of FGFR4 for the treatment of hepatocellular carcinomas with an activated FGFR4 signaling pathway. Cancer Discov. 2015;5(4):424-437.

23. Medina PJ, Goodin S. Lapatinib: a dual inhibitor of human epidermal growth factor receptor tyrosine kinases. Clin Ther. 2008;30(8):1426-1447.

24. Prat A, et al. Prognostic value of intrinsic subtypes in hormone receptor-positive metastatic breast cancer treated with letrozole with or without lapatinib. JAMA Oncol. 2016;2(10):1287-1294.

25. Usary J, Darr DB, Pfefferle AD, Perou CM. Overview of genetically engineered mouse models of distinct breast cancer subtypes. Curr Protoc Pharmacol. 2016;72:14.38.1-14.38.11.

26. Roberts PJ, et al. Combined PI3K/mTOR and MEK inhibition provides broad antitumor activity in faithful murine cancer models. Clin Cancer Res. 2012;18(19):5290-5303.

27. Lim E, et al. Aberrant luminal progenitors as the candidate target population for basal tumor development in BRCA1 mutation carriers. Nat Med. 2009;15(8):907-913.

28. Prat A, Perou CM. Mammary development meets cancer genomics. Nat Med. 2009;15(8):842-844.

29. Prat A, et al. Phenotypic and molecular characterization of the claudin-low intrinsic subtype of breast cancer. Breast Cancer Res. 2010;12(5):R68.

30. Parker JS, et al. Supervised risk predictor of breast cancer based on intrinsic subtypes. J Clin Oncol. 2009;27(8):1160-1167.

31. Tusher VG, Tibshirani R, Chu G. Significance analysis of microarrays applied to the ionizing radiation response. Proc Natl Acad Sci US A. 2001;98(9):5116-5121.

32. Efron B, Tibshirani R, Storey JD, Tusher V. Empirical Bayes analysis of a microarray experiment. J Am Stat Assoc. 2001;96(456):1151-1160.

33. Efron B, Tibshirani R. Empirical bayes methods and false discovery rates for microarrays. Genet Epidemiol. 2002;23(1):70-86.

34. Subramanian A, et al. Gene set enrichment analysis: a knowledge-based approach for interpreting genome-wide expression profiles. Proc Natl Acad Sci U S A. 2005;102(43):15545-15550.
35. Fan C, et al. Building prognostic models for breast cancer patients using clinical variables and hundreds of gene expression signatures. BMC Med Genomics. 2011;4:3.

36. Wallden B, et al. Development and verification of the PAM50-based Prosigna breast cancer gene signature assay. BMC Med Genomics. 2015;8:54.

37. Duncan JS, et al. Dynamic reprogramming of the kinome in response to targeted MEK inhibition in triple-negative breast cancer. Cell. 2012;149(2):307-321.

38. Daub H, et al. Kinase-selective enrichment enables quantitative phosphoproteomics of the kinome across the cell cycle. Mol Cell. 2008;31(3):438-448.

39. Bantscheff M, et al. Quantitative chemical proteomics reveals mechanisms of action of clinical ABL kinase inhibitors. Nat Biotechnol. 2007;25(9):1035-1044.

40. Roidl A, et al. The FGFR4 Y367C mutant is a dominant oncogene in MDA-MB453 breast cancer cells. Oncogene. 2010;29(10):1543-1552.

41. Levine KM, et al. FGFR4 overexpression and hotspot mutations in metastatic $\mathrm{ER}^{+}$breast cancer are enriched in the lobular subtype. NPJ Breast Cancer. 2019;5:19.

42. McFarland JM, et al. Improved estimation of cancer dependencies from large-scale RNAi screens using model-based normalization and data integration. Nat Commun. 2018;9(1):4610.

43. Liberzon A, Birger C, Thorvaldsdóttir H, Ghand M, Mesirov JP, Tamayo P. The Molecular Signatures Database (MSigDB) hallmark gene set collection. Cell Syst. 2015;1(6):417-425.

44. Liberzon A, Subramanian A, Pinchback R, Thorvaldsdóttir H, Tamayo P, Mesirov JP. Molecular signatures database (MSigDB) 3.0. Bioinformatics. 2011;27(12):1739-1740.

45. Tirosh I, et al. Dissecting the multicellular ecosystem of metastatic melanoma by single-cell RNA-seq. Science. 2016;352(6282):189-196.

46. Kim S, Lee K, Choi JH, Ringstad N, Dynlacht BD. Nek2 activation of Kif24 ensures cilium disassembly during the cell cycle. Nat Commun. 2015;6:8087.

47. Pugacheva EN, Jablonski SA, Hartman TR, Henske EP, Golemis EA. HEF1-dependent Aurora A activation induces disassembly of the primary cilium. Cell. 2007;129(7):1351-1363.

48. Jacoby M, et al. INPP5E mutations cause primary cilium signaling defects, ciliary instability and ciliopathies in human and mouse. Nat Genet. 2009;41(9):1027-1031.

49. Ciriello G, et al. Comprehensive molecular portraits of invasive lobular breast cancer. Cell. 2015;163(2):506-519.

50. Cross SS, Hamdy FC, Deloulme JC, Rehman I. Expression of $\mathrm{S} 100$ proteins in normal human tissues and common cancers using tissue microarrays: S100A6, S100A8, S100A9 and S100A11 are all overexpressed in common can- 
cers. Histopathology. 2005;46(3):256-269.

51. Bao YI, Wang A, Mo J. S100A8/A9 is associated with estrogen receptor loss in breast cancer. Oncol Lett. 2016;11(3):1936-1942.

52. Rodriguez-Barrueco R, et al. Inhibition of the autocrine IL-6-JAK2-STAT3-calprotectin axis as targeted therapy for HR-/HER2+ breast cancers. Genes Dev. 2015;29(15):1631-1648.

53. Bergenfelz C, et al. S100A9 expressed in ER(-) $\operatorname{PgR}(-)$ breast cancers induces inflammatory cytokines and is associated with an impaired overall survival. Br J Cancer. 2015;113(8):1234-1243.

54. Welte $\mathrm{T}$, et al. Oncogenic mTOR signalling recruits myeloid-derived suppressor cells to promote tumour initiation. Nat Cell Biol. 2016;18(6):632-644.

55. Gebhardt C, Németh J, Angel P, Hess J. S100A8 and S100A9 in inflammation and cancer. Biochem Pharmacol. 2006;72(11):1622-1631.

56. Steinbakk M, Naess-Andresen CF, Lingaas E, Dale I, Brandtzaeg P, Fagerhol MK. Antimicrobial actions of calcium binding leucocyte L1 protein, calprotectin. Lancet. 1990;336(8718):763-765.

57. D'Amato NC, et al. A TDO2-AhR signaling axis facilitates anoikis resistance and metastasis in triple-negative breast cancer. Cancer Res. 2015;75(21):4651-4664.

58. Novikov O, et al. An aryl hydrocarbon receptormediated amplification loop that enforces cell migration in ER-/PR-/Her2- human breast cancer cells. Mol Pharmacol. 2016;90(5):674-688.

59. Gatza ML, Silva GO, Parker JS, Fan C, Perou CM. An integrated genomics approach identifies drivers of proliferation in luminal-subtype human breast cancer. Nat Genet. 2014;46(10):1051-1059.

60. Rőszer T. Understanding the mysterious M2 macrophage through activation markers and effector mechanisms. Mediators Inflamm. 2015;2015:816460.

61. Duluc D, et al. Tumor-associated leukemia inhibitory factor and IL-6 skew monocyte differentiation into tumor-associated macrophage-like cells. Blood. 2007;110(13):4319-4330.

62. Georgoudaki AM, et al. Reprogramming tumor-associated macrophages by antibody targeting inhibits cancer progression and metastasis. Cell Rep. 2016;15(9):2000-2011.

63. Razavi P, et al. The genomic landscape of endocrine-resistant advanced breast cancers. Cancer Cell. 2018;34(3):427-438.e6.

64. Hoadley KA, et al. Cell-of-origin patterns dominate the molecular classification of 10,000 tumors from 33 types of cancer. Cell. 2018;173(2):291-304.e6.

65. Lefebvre C, et al. Mutational profile of metastatic breast cancers: a retrospective analysis. PLoS Med. 2016;13(12):e1002201.

66. Forbes SA, et al. COSMIC: somatic cancer genetics at high-resolution. Nucleic Acids Res. 2017;45(D1):D777-D783.

67. Catalogue of somatic mutations in cancer (COSMIC). https://www.cancer.sanger.ac.uk. Updated April 7, 2020. Accessed June 26, 2020.

68. Siegel MB, et al. Integrated RNA and DNA sequencing reveals early drivers of metastatic breast cancer. J Clin Invest. 2018;128(4):1371-1383.

69. Harrell JC, et al. Genomic analysis identifies unique signatures predictive of brain, lung, and liver relapse. Breast Cancer Res Treat. 2012;132(2):523-535.

70. Smid M, et al. Subtypes of breast cancer show preferential site of relapse. Cancer Res. 2008;68(9):3108-3114.

71. Sihto H, et al. Breast cancer biological subtypes and protein expression predict for the preferential distant metastasis sites: a nationwide cohort study. Breast Cancer Res. 2011;13(5):R87.

72. Gao G, Goldfarb M. Heparin can activate a receptor tyrosine kinase. $E M B O J .1995 ; 14(10): 2183-2190$.

73. Loo BM, Kreuger J, Jalkanen M, Lindahl U, Salmivirta M. Binding of heparin/heparan sulfate to fibroblast growth factor receptor 4.J Biol Chem. 2001;276(20):16868-16876.

74. Cavallaro U, Niedermeyer J, Fuxa M, Christofori G. N-CAM modulates tumour-cell adhesion to matrix by inducing FGF-receptor signalling. Nat Cell Biol. 2001;3(7):650-657.

75. Pan H, et al. 20-Year risks of breast-cancer recur- rence after stopping endocrine therapy at 5 years. N Engl JMed. 2017;377(19):1836-1846.

76. Kennecke H, et al. Metastatic behavior of breast cancer subtypes. J Clin Oncol. 2010;28(20):3271-3277.

77. Saraiva DP, Guadalupe Cabral M, Jacinto A, Braga S. How many diseases is triple negative breast cancer: the protagonism of the immune microenvironment. ESMO Open. 2017;2(4):e000208.

78. Ogiya R, et al. Comparison of tumor-infiltrating lymphocytes between primary and metastatic tumors in breast cancer patients. Cancer Sci. 2016;107(12):1730-1735.

79. Sobottka B, Pestalozzi B, Fink D, Moch H, Varga Z. Similar lymphocytic infiltration pattern in primary breast cancer and their corresponding distant metastases. Oncoimmunology. 2016;5(6):e1153208.

80. Szekely B, et al. Immunological differences between primary and metastatic breast cancer. Ann Oncol. 2018;29(11):2232-2239.

81. Zhu L, et al. Metastatic breast cancers have reduced immune cell recruitment but harbor increased macrophages relative to their matched primary tumors. J Immunother Cancer. 2019;7(1):265.

82. Meijer D, Sieuwerts AM, Look MP, van Agthoven T, Foekens JA, Dorssers LC. Fibroblast growth factor receptor 4 predicts failure on tamoxifen therapy in patients with recurrent breast cancer. Endocr Relat Cancer. 2008;15(1):101-111.

83. Clinical Trials - A service of the US National Institutes of Health. http://clinicaltrialsgov. 2018. NCT03238196, NCT03344536.

84. Mermel CH, Schumacher SE, Hill B, Meyerson ML, Beroukhim R, Getz G. GISTIC2.0 facilitates sensitive and confident localization of the targets of focal somatic copy-number alteration in human cancers. Genome Biol. 2011;12(4):R41.

85. Cancer Genome Atlas Network. Comprehensive molecular portraits of human breast tumours. Nature. 2012;490(7418):61-70. 